\title{
Effects of multiple phase transitions in a three- dimensional spherical model of convection in Earth's mantle
}

\author{
Paul J. Tackley, ${ }^{1}$ David J. Stevenson, ${ }^{2}$ Gary A. Glatzmaier, ${ }^{3}$ and Gerald Schubert ${ }^{4}$
}

Abstract. Numerical models of mantle convection that incorporate the major mantle phase changes of the transition zone reveal an inherently three-dimensional flow pattern, with cylindrical features and linear features that behave differently in their ability to penetrate the 670km discontinuity. The dynamics are dominated by accumulation of cold linear downwellings into rounded pools above the endothermic phase change at $670 \mathrm{~km}$ depth, resulting in frequent "avalanches" of upper mantle material into the lower mantle. The effect of the exothermic phase transition at $400 \mathrm{~km}$ depth is to reduce the overall degree of layering by pushing material through the 670-km phase change, resulting in smaller and more frequent avalanches, and a wider range of morphologies. Large quantities of avalanched cold material accumulate above the coremantle boundary (CMB), resulting in a region of strongly depressed mean temperature at the base of the mantle. The 670-km phase change has a strong effect on the temperature field, with three distinct regions being visible: (1) the upper mantle, containing linear downwellings and pools of cold material in the transition zone and characterized by a high amplitude long-wavelength spectrum; (2) the midmantle, containing quasi-cylindrical avalanche conduits and characterized by a low amplitude, broad spectrum; and (3) the deep mantle, containing large pools of cold, avalanched material and characterized by a high amplitude, ultra-red (i.e., long wavelength) spectrum. The effect on the velocity field is very different. Flow penetration across the 670-km phase change is strongly wavelength-dependent, with easy penetration at long wavelengths but strong inhibition at short wavelengths. Thus, when comparing numerical models with longwavelength seismic tomography, diagnostics based on the density field, such as the radial correlation function, are much more sensitive to the effects of phase transitions than those based on the velocity field. The amplitude of the geoid is not significantly affected by the partial layering, because the contribution from the strong heterogeneity in the transition zone is almost perfectly balanced by the contribution from deflection of the 670-km discontinuity. Avalanches are associated with geoid lows. However, a more complex viscosity structure is required to correctly match the sign of the geoid over slabs in Earth.

\section{Introduction}

The major seismic discontinuities at depths variously described as $400-440 \mathrm{~km}$ and $650-700 \mathrm{~km}$ bound the transition zone and are now widely accepted as primarily representing mineralogical phase transformations [Birch, 1952; Ringwood, 1982, 1991], although associated and perhaps induced chemical changes at around the same depths remain an intriguing possibility [Anderson, 1989; Jeanloz, 1991; Ringwood, 1991; Weinstein, 1992].

The lower of these transitions, associated with the phase change from $\gamma$-spinel to perovskite + magnesiowüstite at

\footnotetext{
${ }^{1}$ Seismological Laboratory, California Institute of Technology, Pasadena.

${ }^{2}$ Division of Geological and Planetary Sciences, California Institute of Technology, Pasadena.

${ }^{3}$ Institute of Geophysics and Planetary Physics, Los Alamos National Laboratory, Los Alamos, New Mexico.

${ }^{4}$ Department of Earth and Space Sciences and Institute of Geophysics and Planetary Physics, University of California, Los Angeles.

Copyright 1994 by the American Geophysical Union.

Paper number 94JB00853.

0148-0227/94/94JB-00853505.00
}

around $670 \mathrm{~km}$ depth, is endothermic and therefore resists the flow of material across it [Schubert et al., 1975]. The question of whether this phase transition could enforce layered convection in Earth's mantle has received much attention and has profound implications for Earth's thermal and chemical structure and evolution [e.g., Wyllie, 1988]. Early two-dimensional (2-D) numerical modeling [Christensen and Yuen, 1985] indicated that an unrealistically large Clapeyron slope of $-6 \mathrm{MPa} \mathrm{K}^{-1}$ would be required for layering to occur. However, recent work in two-dimensional Cartesian [Liu et al., 1991; Zhao et al., 1992; Weinstein, 1993] and spherical axisymmetric [Machetel and Weber, 1991; Peltier and Solheim, 1992] geometries with realistic phase change parameters bas indicated significant layering, as well as complex new phenomena and modes of time dependence in the flow. In particular, Peltier and Solheim [1992] and Weinstein [1993] find periods of strong layering punctuated by brief, vigorous episodes of whole mantle flow associated with catastrophic "avalanches".

The additional influence exerted by the other major phase transition at around $400 \mathrm{~km}$ depth, associated with the phase change from olivine to B-spinel, which is exothermic and therefore enhances the flow of material across it [Schubert et al., 1975], has been included in many of these studies. Zhao et al. [1992] and Steinbach and Yuen [1992] demonstrate its ef- 
fect on introducing another dynamical timescale to the system. However, the question of whether its influence increases or decreases the overall propensity to layering is unclear, since different studies show conflicting results on this issue [Steinbach and Yuen, 1992; Zhao et al., 1992; Solheim and Peltier, 1994].

In order to understand Earth, it is essential to determine how the effects observed in these two-dimensional studies are modified in three-dimensional (3-D) geometry. Tackley et al. [1993] presented a simulation performed in three-dimensional spherical geometry with the endothermic phase change at 670 $\mathbf{k m}$ depth and heat flow approximately a factor of 2 lower than that characteristic of Earth, suggesting a Rayleigh number too low by almost an order of magnitude (assuming $N u \propto R a_{T}{ }^{1 / 3}$ [Turcotte and Schubert, 1982]) and found that the avalanches, or "flushing events" occurred exclusively in cylindrical forms and that the time dependence of global quantities was weak. These results were corroborated by the calculation of Honda $e t$ al. [1993] in a completely basally heated Cartesian box with two phase changes present and at a similar Rayleigh number. More recent results by Yuen et al. [1994] in the same geometry demonstrate the effect of higher Rayleigh number in reducing the mass flux across the $670-\mathrm{km}$ discontinuity, which was previously demonstrated in two dimensions by Christensen and Yuen [1985].

Although such 3-D calculations still lack many of the features of the real Earth, such as plates, they are progressively becoming more "realistic", and thus it is desirable to fully understand and analyze their implications for mantle dynamics and to devise ways of comparing them to actual observations of Earth, such as seismic tomographic models and the geoid. By understanding and quantifying the strengths and weaknesses of current convection models in these comparisons, we can establish a reference point for future, perhaps more realistic, studies. For this purpose, the use of spherical geometry is clearly an important ingredient, as it facilitates direct comparison with Earth observations and has previously been found to significantly modify the heat flow characteristics, geometry of lower mantle flow, and upwelling plume structure [Bercovici et al., 1989; Tackley et al., 1993]. Thus, in this paper we extend the analysis of the result reported by Tackley et al. [1993] and compare this with new simulations which contain the $400-\mathrm{km}$ phase change, as well as with observations of Earth, including seismic tomographic models. The geoid has not been considered in any of the numerical results referenced above, yet is one of the most important constraints on the internal structure and dynamics [Kaula, 1989]. Thus we calculate the geoid for these cases and characterize its amplitude spectrum and relationship to internal processes in our model. The spatial resolution and Rayleigh number of these convection models are much higher than in previous spherical models with no phase change [Baumgardner, 1985, 1988; Bercovici et al., 1989; Glatzmaier et al., 1990; Schubert et al., 1990].

\section{Model, Method, and Parameters}

\section{Overview}

Our physical model and numerical method are modified versions of those described by Glatzmaier [1988]. The equations of compressible self-gravitating flow are solved and integrated in time using a spectral transform method, implemented on the
Intel Touchstone Delta parallel supercomputer system at the California Institute of Technology. Since the mantle is characterized by very high Prandtl number, inertial terms are ignored. The anelastic approximation is used, eliminating acoustic waves which propagate many orders of magnitude faster than convective velocities. The state of the system is expressed as the combination of a time-independent, radially dependent adiabatic reference state (discussed later), and the time-dependent three-dimensional variables of poloidal mass flux potential, and perturbations to entropy, pressure, and gravitational potential fields. The time-dependent variables are expanded laterally in spherical harmonics, up to degree and order 127, and the nonlinear products associated with advection and viscous dissipation are evaluated in grid space using 384 longitudinal points by 192 latitudinal points. Variables are expanded vertically in Chebyshev series. For the cases with two phase changes, three separate expansions are used, corresponding to the lower mantle (33 radial levels), transition zone (13 radial levels), and shallow mantle (13 radial levels). For the case with a single phase change, two expansions are used with 33 lower mantle and 17 upper mantle levels, and for whole mantle cases, a single expansion of 41 radial levels is used. Use of multiple Chebyshev expansions matched at the phase change depths gives excellent vertical resolution ( -5 $\mathrm{km}$ ) at the core-mantle boundary (CMB), phase change depths, and the surface, where it is most needed. Adjacent Chebyshev expansions are matched at the internal interfaces by continuity of temperature, conductive heat flux, mass flux, tangential velocity and tangential stress, with a discontinuity in normal stress and gravitational acceleration, as explained in the next section. External boundaries (surface and CMB) are stress-free and isothermal. The fields are adequately resolved since the horizontal and vertical variances of variables fall by many orders of magnitude between maximum values and truncation [Glatzmaier, 1988; Bercovici et al., 1989; Schubert et al., 1990].

\section{Phase Change Parameterization}

Our model incorporates the effects of the exothermic olivine to B-spinel transition occurring at around $400 \mathrm{~km}$ depth (hereafter referred to as the 400), and endothermic $\gamma$ spinel to perovskite plus magnesiowustite transition occurring at around $670 \mathrm{~km}$ depth (hereafter referred to as the 670), consistent with a peridotitic mantle composition [Ito and Takahashi, 1989].

Since the phase boundary deflection observed in Earth [Shearer and Masters, 1992] is at least an order of magnitude smaller than the characteristic size of convective features in our model, it is not necessary (to a good approximation) to resolve the exact details of the phase change. A posteriori justification of this assumption is provided by the actual calculated phase boundary deflections. Thus deflection of the phase change resulting from lateral temperature variations at 400 or $670 \mathrm{~km}$ depth is represented as a sheet mass anomaly at that depth, resulting in discontinuous normal stress and gravitational acceleration between two adjacent Chebyshev regimes. This approach has the advantage of a zero-width phase loop (the pressure or depth interval over which the multivariant phase change occurs), important since the phase loop width significantly affects the inhibition of flow across the boundary [Peltier and Solheim, 1992], and recent laboratory experiments [Ito and Takahashi, 1989] and seismological observations [Benz and Vidale, 1993] constrain this width at $670 \mathrm{~km}$ 
to be a few kilometers at most. This method is equivalent to the "effective thermal expansivity" approach [Christensen and Yuen, 1985], collapsed into a discontinuity. For numerical reasons, the latent heat release (absorption) that accompanies upward (downward) motion through the phase change must be spread out $25 \mathrm{~km}$ on either side of the interface. Latent heat release has a very small effect on the propensity to layering, as shown by Christensen and Yuen [1985]; thus the details of its implementation are probably not critical to the model results. This treatment of the phase change has been validated by computing two-dimensional Cartesian and spherical axisymmetric results and comparing to previously published results in which the phase boundary deflection is fully resolved [Christensen and Yuen, 1985; Machetel and Weber, 1991]. These tests confirm that this approximation is reasonable, giving the right magnitude of the effect, at the Rayleigh numbers presented here. Additional tests suggest that at higher Rayleigh numbers, where peak-to-peak phase boundary deflection is comparable to the width of convective features, both this technique and the effective thermal expansivity technique may overestimate the effect of the phase changes.

\section{Gravitational Field}

Although perturbations in the gravitational field have a negligible effect on the time evolution of the flow [Glatzmaier, 1988], we include them in order to study the geoid, which is one of the most important geophysical observables, providing clues as to processes in the interior [Kaula, 1989]. The net geoid is a balance between two opposite and nearly equal contributions: (1) internal mass anomalies and (2) dynamic surface and CMB topography [Ricard et al., 1984; Richards and Hager, 1984]. Since the resulting gravitational perturbation acts in such a way as to produce further surface deformation (a self-gravitational effect which is very important at long wavelengths), it is essential to calculate gravitational perturbation simultaneously with the flow field. We do this, using a first-order treatment of surface and CMB dynamic topography [Hager and Clayton, 1989], in which the deformation is treated as a sheet mass anomaly causing a discontinuity in vertical gradient of gravitational potential over the boundary in question. Deflections of the phase changes due to temperature perturbations are treated in a similar manner. The external and internal fields are constrained to be bounded solutions [Glatzmaier, 1988]. This implementation was checked by computing geoid kernels for sheet mass anomalies at various depths and comparing to published kernels calculated using a propagator matrix technique [Hager and Clayton, 1989].

\section{Reference State}

Entropy, pressure, and gravitational potential are expanded as perturbations relative to a self-gravitating adiabatic reference state. The Murnaghan equation is assumed, leading to a polytrope [Glatzmaier, 1988] (i.e., $P \propto\left[\rho^{1+1 / n}-\rho_{0}^{1+1 / n}\right]$, where $n$ is the polytropic index, $\rho$ is the density, and $\rho_{0}$ is the zero-pressure density). Implicit in this treatment is the depth dependence of material properties such as density, bulk modulus, and thermal expansivity but with the Gruneisen parameter and heat capacity kept constant. Thermal conductivity and viscosity are specified as functions of depth only. Parameter values are given in Table 1 . The parameters were chosen to give a reasonable fit to the Preliminary Reference Earth Model
Table 1. Reference State Parameters

\begin{tabular}{|c|c|c|c|}
\hline Parameters & $\begin{array}{l}\text { Two-Layer } \\
\text { Cases }\end{array}$ & $\begin{array}{c}\text { Three-Layer } \\
\text { Cases }\end{array}$ & Units \\
\hline $\begin{array}{l}\text { Gruneisen } \\
\text { Polytropic n } \\
\text { Heat capacity } \\
\rho \text {, surface } \\
\rho, \text { CMB } \\
\alpha, \text { surface } \\
\alpha, 670 \text { km } \\
\alpha, \text { CMB } \\
\eta \text {, surface } \\
\eta, 670 \text { km } \\
\eta, \text { CMB } \\
k \text {, surface } \\
\text { k, CMB } \\
\text { Tsurface } \\
\text { Tcmb } \\
\Delta \text { Tsa } \\
\text { Qint } \\
\text { Ra } \\
\text { Rat } \\
\text { Ra } \\
\text { T,surf }\end{array}$ & $\begin{array}{c}1.0 \\
0.5 \\
1250 \\
3610 \\
5600 \\
3.0 \times 10^{-5} \\
2.2 \times 10^{-5} \\
1.2 \times 10^{-5} \\
1.7 \times 10^{22} \\
1.9 \times 10^{22} \\
2.1 \times 10^{23} \\
2.4 \\
14.0 \\
1060 \\
3450 \\
1250 \\
2.0 \times 10^{-12} \\
1.2 \times 10^{6} \\
1.8 \times 10^{7} \\
3.5 \times 10^{6}\end{array}$ & $\begin{array}{c}1.0 \\
0.4 \\
1250 \\
3610 \\
5600 \\
3.3 \times 10^{-5} \\
2.3 \times 10^{-5} \\
1.1 \times 10^{-5} \\
1.8 \times 10^{22} 2^{*} \\
2.1 \times 10^{22} * \\
2.1 \times 10^{23 *} \\
2.4 \\
14.0 \\
940 \\
3150 \\
1250 \\
2.0 \times 10^{-12} \\
1.2 \times 10^{6} \\
1.8 \times 10^{7} \\
3.7 \times 10^{6}\end{array}$ & 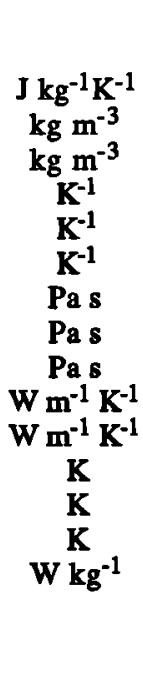 \\
\hline
\end{tabular}

Case 5 has a constant kinematic viscosity of $4.7 \times 10^{16} \mathrm{~m}^{2}$ $\mathrm{s}^{-1}$, giving a dynamic viscosity of $1.7 \times 10^{22}$ (surface) to $2.6 \times 10^{22}$ (CMB) Pa s.

(PREM) [Dziewonski and Anderson, 1981] and were improved when the $400-\mathrm{km}$ phase change was added, resulting in two different, though fairly similar, sets of parameters.

The fractional decrease of thermal expansivity over the lower mantle (about 2.1) is reasonably close to the factor of 2.5 found in recent thermodynamic analyses [Chopelas and Boehler, 1992; Anderson et al., 1992; Duffy and Ahrens, 1993]. Thermal conductivity increases with depth as the fourth power of density, giving a lower mantle increase consistent with experiments [Osako and Ito, 1991] and theory [Anderson, 1987].

However, the uncertainties in the radial profile of viscosity are much more significant than uncertainties in the exact depth dependencies of such thermodynamic parameters, and the assumption that the viscosity is constant horizontally is perhaps the most significant. We make the latter assumption to keep the computational task reasonable, since time-dependent 3-D simulations with strongly temperature-dependent viscosity require considerable computational resources and are only recently becoming feasible [Tackley, 1993]. There is general agreement in the literature for a viscosity increase with depth across the mantle [Hager and Richards, 1989; Ricard and Wuming, 1991; King and Masters, 1992; Forte et al., 1993; Mitrovica and Peltier, 1993]. However, the magnitude and exact form of this increase are highly uncertain, since there is a considerable null space associated with current data sets and inversion techniques (S.D. King, Radial models of mantle viscosity: Results from a genetic algorithm, submitted to Geophys. J. Int., 1994). For this study we arbitrarily specify a smooth, power law dependence of viscous diffusivity on density (giving a depth profile similar to an exponential dependence on depth) with an index of zero in the upper mantle (constant viscous diffusivity) and 7 in the lower mantle, resulting in an increase of dynamic viscosity by a factor of -12 over the depth of the mantle. Values of dynamic viscosity at the surface, $670 \mathrm{~km}$ depth, and $\mathrm{CMB}$ are given in Table 1. 


\section{Thermal Parameters}

Surface and CMB temperatures and internal heating rates are also listed in Table 1 . Since our formulation lacks rigid plates, the surface temperature is chosen to represent the base of the lithosphere (as by Bercovici et al. [1989], Glatzmaier et al. [1990], and Machetel and Weber [1991]). However, absolute temperatures in such a calculation are not meaningful except to the extent that they are adjusted to be realistic at some level (e.g., the upper mantle); only temperature differences are relevant to the convective style and vigor. Since the ratio of internal heating to basal heating strongly affects the degree of layering in two-dimensional models [Solheim and Peltier, 1994], it is important to obtain a ratio similar to that in Earth. Thus, because our superadiabatic temperature drop is lower than that of Earth, as explained above, we reduce the chondritic heating rate [Stacey, 1992] proportionally in order to maintain a ratio of internal to basal heating similar to that commonly believed, although this ratio is not well known [Leitch and Yuen, 1989].

The volume-averaged Rayleigh numbers resulting from internal heating $\left(R a_{H}\right)$ and superadiabaticity $\left(R a_{T}\right)$, as defined below, are given in Table 1:

$$
\begin{aligned}
R a_{T} & =\left\langle\frac{\bar{g}(r) \bar{\alpha}(r)}{\bar{v}(r) \bar{\kappa}(r)}\right\rangle \Delta T_{s e} d^{3} \\
R a_{H} & =\left\langle\frac{\bar{g}(r) \bar{\alpha}(r)}{\bar{v}(r) \bar{\kappa}(r) \bar{k}(r)}\right\rangle Q d^{5}
\end{aligned}
$$

where over bars denote quantities that are the radially dependent reference state quantities thermal expansivity $\alpha$, gravitational acceleration $g$, viscous diffusivity $v$, thermal diffusivity $\kappa$, and thermal conductivity $\left(k=\rho c_{p} \kappa\right) ; \Delta T_{s a}$ is the superadiabatic temperature drop; $Q$ is the internal heating rate per unit mass; and $d$ is the depth of the mantle. Table 1 also lists $R a_{\text {surf }}$, the surface Rayleigh number used by some authors [e.g., Honda et al., 1993], which is defined as $R a_{T}$ above but with the volume average being replaced by values at the surface. These Rayleigh numbers are about an order of magnitude higher than in previous spherical studies [Glatzmaier et al., 1990] but almost an order of magnitude less than those characterizing Earth. This is due to computational limitations: higher Rayleigh numbers would result in narrower features which could not be adequately resolved at the highest numerical resolution currently practical. Realistic Rayleigh numbers are obtainable in 2-D calculations [Liu et al., 1991; Zhao et al., 1992; Peltier and Solheim, 1992], and in 3-D Cartesian calculations [Yuen et al., 1994], which might be useful for predicting how the effects observed here might scale to the Earth-like regime.

\section{Phase Change Parameters}

There has been a tendency in studies of convection with phase transitions to focus on the Clapeyron slope as the only important parameter; however, this is misleading since the effectiveness of a phase transition in inhibiting or assisting flow, due to the anomalous buoyancy associated with its vertical deflection, is given by a nondimensional combination of parameters known as the phase buoyancy parameter $P$ [Schubert and Turcotte, 1971; Christensen and Yuen, 1985]:

$$
P=\frac{\gamma \Delta \rho}{\alpha \rho^{2} g h}
$$

where $\gamma$ is the Clapeyron slope, $\Delta p$ is the density change associated with the phase transition, $\alpha$ is the thermal expansivity, $\rho$ is the background density, $g$ is the gravitational acceleration, and $h$ is a depth scale for the convection, here taken to be the depth of the mantle. Thus the magnitude of the density jump across the phase change and the local thermal expansivity at the phase change depth are as important as the Clapeyron slope, and all three parameters are subject to some uncertainty.

The $670-\mathrm{km}$ phase transition has a density jump of $10-11 \%$ and a Clapeyron slope which is given by laboratory experi-

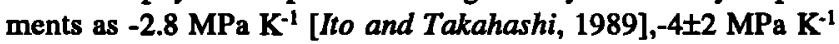
[Ito et al., 1990], or $-3 \pm 1 \mathrm{MPa} \mathrm{K}^{-1}$ [Akaogi and Ito, 1993], compatible with the value of -1.7 to $-4.4 \mathrm{MPa} \mathrm{K}^{-1}$ calculated by Wicks and Richards [1993] by combining seismic observations of 670 deflection beneath the Izu-Bonin subduction zone with a thermal model of the subducted slab. The $400-\mathrm{km}$ transition has a lower density jump given as 5\% in PREM [Dziewonski and Anderson, 1981] or $8 \%$ in recent mineralogical studies [Akaogi et al., 1989; Katsura and Ito, 1989], and a Clapeyron slope in the range $1.5 \mathrm{MPa} \mathrm{K}^{-1}$ [Akaogi et al., 1989] to $2.5 \mathrm{MPa} \mathrm{K}^{-1}$ [Katsura and Ito, 1989]. Thus the magnitude of $\boldsymbol{P}_{400}$ is much smaller than the magnitude of $\boldsymbol{P}_{670}$, since both the density jump and Clapeyron slope are of lower magnitude, and the thermal expansivity is larger at $400 \mathrm{~km}$ depth than at $670 \mathrm{~km}$ depth [Chopelas and Boehler, 1992; Anderson et al., 1992]. However, there is some uncertainty about the relative importance of the 400 and 670 since the phase transitions are not equidistant from all boundaries, which may affect the best choice of length scale $h$ in equation (2).

The latent heat release or absorption $Q_{L}$ associated with a phase transition is related to the other parameters by:

$$
Q_{L}=\frac{\gamma T \Delta \rho}{\rho^{2}}
$$

where $T$ is the temperature and the other symbols are defined above.

\section{Simulation Details}

Five simulations are presented here, using four different sets of phase change parameters, as listed in Table 2, which lists the phase buoyancy parameters $P_{400}$ and $P_{670}$ as well as the dimensional Clapeyron slopes and density jumps. For the 670 , we take the preferred values of density change and Clapeyron slope from Ito et al. [1990], except for case 4, in which we test the sensitivity of the system to $\gamma_{670}$ by reducing it to $-3 \mathrm{MPa}$ $\mathrm{K}^{-1}$. In cases 1 to 3 , the strength of the 400 is increased from

Table 2. Phase Change Parameters

\begin{tabular}{lcccc}
\hline & \multicolumn{5}{c}{ Case } \\
\cline { 2 - 5 } & 1 & 2,5 & 3 & 4 \\
\hline$\gamma_{400}, \mathrm{MPa} \mathrm{K}^{-1}$ & 0 & 3.0 & 4.0 & 3.0 \\
$\delta \rho_{400}, \mathrm{~kg} \mathrm{~m}^{-3}$ & 0 & 200.0 & 400.0 & 200.0 \\
$\gamma_{670}, \mathrm{MPa} \mathrm{K}^{-1}$ & -4.0 & -4.0 & -4.0 & -3.0 \\
$\delta \rho_{670}, \mathrm{~kg} \mathrm{~m}^{-3}$ & 400.0 & 400.0 & 400.0 & 400.0 \\
$\mathbf{P}_{400}$ & 0.0 & 0.055 & 0.135 & 0.055 \\
$\mathbf{P}_{670}$ & -0.147 & -0.147 & -0.147 & -0.110 \\
\hline
\end{tabular}


zero to a value much larger than that likely for the mantle, in order to assess its influence on the convective system. Case 5 is as case 2 except with a constant viscous diffusivity throughout the mantle, instead of a smooth viscosity increase with depth.

Although a value of $-4 \mathrm{MPa} \mathrm{K} \mathbf{K}^{-1}$ for $\boldsymbol{\gamma}_{670}$ has been observed to cause very strong layering in one axisymmetric result [Machetel and Weber, 1991], the inhibiting effect of the phase change in that simulation was greatly enhanced by the use of a very much lower thermal expansivity at $670 \mathrm{~km}$ depth $\left(1.4 \times 10^{-5} \mathrm{~K}^{-1}\right)$ than is consistent with experimental and theoretical estimates [Chopelas and Boehler, 1992; Anderson et al., 1992; Duffy and Ahrens, 1993] resulting in a very low $P_{670}$ of around -0.22 .

Case 1 was started from a case with no phase change and run for about 15,000 time steps, corresponding to about 3 b.y.; the results we present here are characteristic of the last 9000 time steps, after the system has overcome the initial transient adjustment to the presence of the phase change. Cases 2-5 were started from final states of other runs with similar parameters and run until the system overcame its transient adjustment to the new parameters, which typically took 510,000 time steps.

\section{Results}

\section{Heat Flow and Velocities}

We obtain mean surface heat flows of around $1.8 \times 10^{13} \mathrm{~W}$, of which $\sim 40 \%$ comes from the core in cases $1-4$, and $-60 \%$ comes from the core in case 5 . The total is about a factor of 2 smaller than that of Earth, but the fraction of basal heating is considerably larger than most estimates for Earth [Schubert, 1979; Schubert et al., 1980; Davies and Richards, 1992]. It is possible that the mantle heating rate should be substantially augmented by the effect of secular cooling [Sharpe and Peltier, 1978; Schubert et al., 1980] which may account for $30 \%$ of the surface heat flow [Breuer and Spohn, 1993]. This is missing from the model because the heating is treated as timeindependent; the effect is only recently receiving attention from the numerical modeling community [Steinbach et al., 1993].

Maximum convective velocities are in the range $25-45 \mathrm{~mm}$ $\mathrm{yr}^{-1}$, depending on the size and vigor of avalanches, and average surface velocities are typically 5-6 $\mathrm{mm} \mathrm{yr}^{-1}$, an order of magnitude lower than plate velocities, though a direct comparison may be flawed because our simulations lack rigid plates. The principal difference between our parameters and Earth probably lies in the use of higher than realistic viscosities, which is necessitated by computing limitations. At the higher Rayleigh numbers which would result from lower viscosities, the phase change has a stronger inhibitive effect on both twodimensional [Christensen and Yuen, 1985; Zhao et al., 1992] and three-dimensional [Yuen et al., 1994] flow; thus our calculation may underpredict the degree of layering which would occur in the Earth-like regime.

\section{Flow Pattern}

Effect of the 670. Plate 1 illustrates cold and hot features for case 1 (with only the 670 included) at three times at intervals of approximately $500 \mathrm{my}$. The basic structure of the flow was described by Tackley et al. [1993]. A network of interconnected cold downwelling sheets, which do not penetrate the phase change, is observed in the upper mantle (UM). The distance between sheets that are roughly parallel is typically $3000-8000 \mathrm{~km}$, a scale which is consistent with present subduction zones on Earth. They have some smallscale complexity due to local boundary layer instabilities, which would likely be suppressed by the high-viscosity lithospheric plates on Earth. At the intersections of these sheets, large pools of cold material form above the $670-\mathrm{km}$ phase change. This cold material is gravitationally unstable and after building up sufficiently triggers a vigorous avalanche into the lower mantle, in the form of a very large diameter $(\sim 1000 \mathrm{~km})$ cylindrical downwelling plume, which reaches the CMB on a timescale of several tens of million years. This downwelling acts as a conduit to the CMB, effectively emptying the cold material from the local upper mantle to a large pool at the base of the mantle, despite the increase in viscosity with depth. The increase in viscosity with depth slows these downflows and results in the region of spreading being of greater vertical extent. The downwelling then shuts off completely and is not observed to recur in exactly the same place during the course of the simulation, though many such events may occur in the same general area (such as the lower left region of these plots), and about 15 events are observed in total during the final 2 b.y. of the simulation. Thus these events cool all areas of the lower mantle and core. At any one time, several of these avalanches are in progress at different places around the sphere, triggering in a globally asynchronous manner. Because of the spherical geometry, the surface area of the CMB is about $35 \%$ of the surface area of the $670-\mathrm{km}$ interface, and thus the combined effect of avalanches occurring in different places around the sphere is to surround the core with cold material, resulting in a $\mathrm{CMB}$ heat flow ( $-40 \%$ of surface heat flow) considerably higher than most estimates for Earth, as discussed above, but with very little upwelling plume activity. Thus most of the core heat flux is taken up in heating up these "dead slabs" rather than feeding active upwelling plumes. This may be in accord with Earth, since Olson et al. [1993] conclude on the basis of numerical simulations of plumes with realistic rheology that the entire mantle plume population draws material from less than $20 \%$ of the core-mantle boundary.

In some areas, where elongated or larger pools of cold material accumulate above the 670 , multiple cylindrical avalanches, closely spaced in time, may occur. This is happening in the bottom left of Plate 1.

The most prominent features in the pattern of hot upwellings are the broad hot regions in the upper mantle, which are often not directly associated with any deep features in the lower mantle. It is apparent that the lateral heterogeneity is much greater in the upper mantle than at midmantle depths, as shown quantitatively later on. At the CMB, interconnected ridges of hot material are observed, which extend radially upward several hundred kilometers. These are swept around by enormous injections of cold upper mantle material caused by avalanches, and occasionally a larger accumulation of hot material is formed at the intersection of these ridges, causing a short-lived, transient plume to rise through the midmantle to the $670-\mathrm{km}$ interface and inject hot material into the upper mantle. One of these can be observed in the upper part of Plate 1f. Thus, in both directions, cylindrical forms (plumes) are seen to penetrate the $670-\mathrm{km}$ phase change, whereas linear forms (sheets) do not. The upward flow in the upper mantle and in the top of the lower 


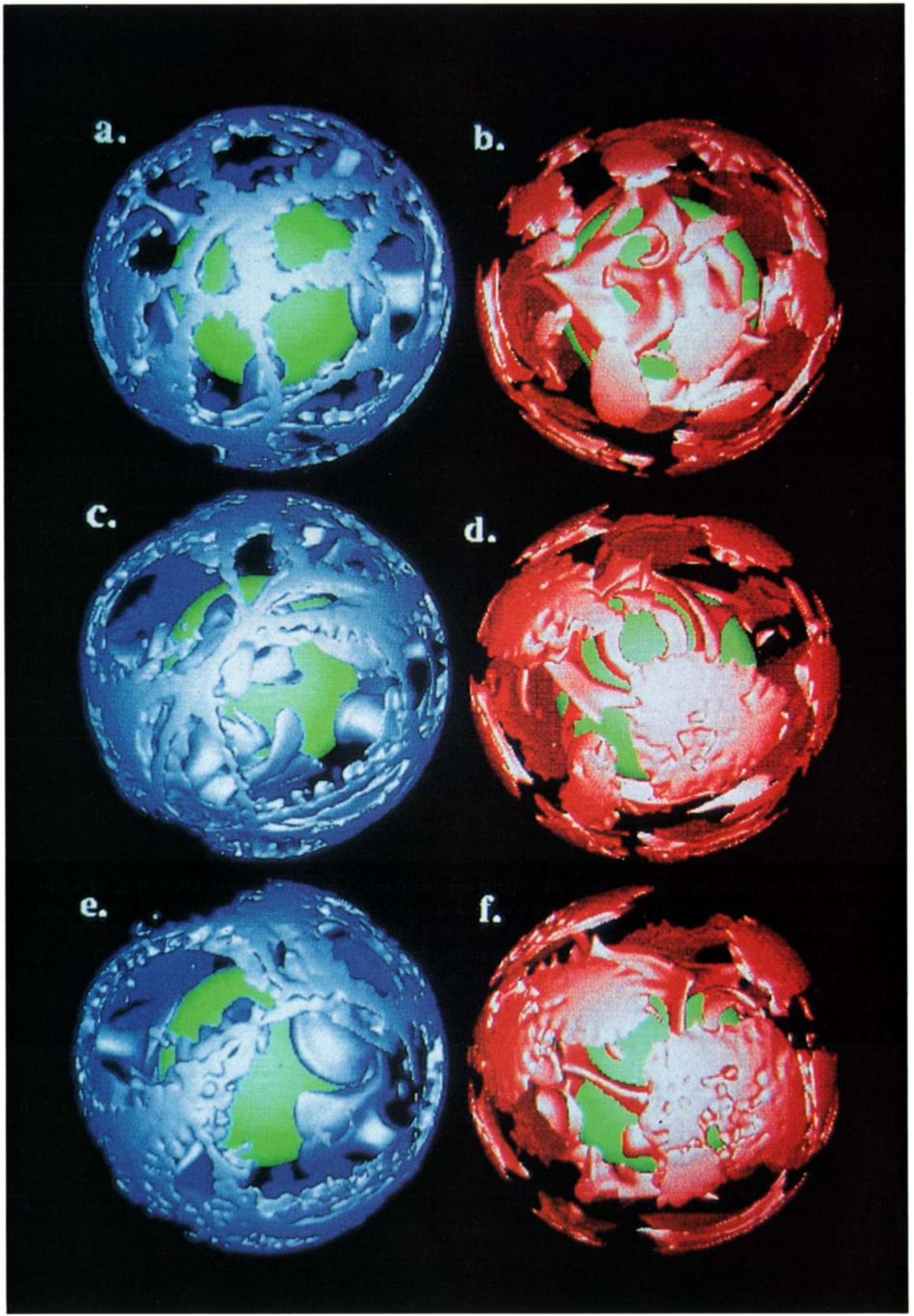

Plate 1. (left) Cold downwellings and (right) hot upwellings for case 1 (single phase change) at three times during the simulation, at intervals of $\sim 500 \mathrm{~m}$.y. Cold and hot isosurfaces show where the temperature is $110 \mathrm{~K}$ lower and higher, respectively, than the horizontal average. The core-mantle boundary is shown in green. Time increases down the figure, with the bottom figures depicting the final state of the simulation. 
mantle is generally the weak, distributed return flow characterizing mainly internally heated convection [Houseman, 1988; Glatzmaier et al., 1990; Travis et al., 1990]. It is interesting that the large hot structures in the deep mantle, which should be quite buoyant, have difficulty ascending through the midmantle. The reason is that although they are hot relative to the spherically averaged temperature in the deep mantle, they are not particularly hot relative to the spherically averaged midmantle temperature, because the temperature in the deep mantle is strongly depressed by the large pools of cold avalanched material.

Effect of adding the 400 . Plate 2 shows similar views for case 3 (with a 400 of approximately equal strength to the 670), at three times at intervals of 500 m.y. Here the flow is more complex. Avalanches display a wider range of morphologies, including quasi-linear, and are generally smaller and more frequent. This is because the 400 limits the extent to which cold material can accumulate above the 670: when accumulations become thick enough to straddle the 400 , the additional negative buoyancy caused by the upward deflection of the $400-\mathrm{km}$ phase change pushes the cold material through the 670 , resulting in more frequent and smaller avalanches. Thus, it appears that the effectiveness of the 670 on inhibiting mass flux between the upper and lower mantles is reduced by the 400 , a point which is shown quantitatively later. A range of downflow morphologies occurs because such breakthroughs may be forced before cold material is able to flow to triple junctions and accumulate into broad pools.

Several strong, stable plumelike upwellings, which penetrate the 670 with ease, are visible. These form in this case partly because the smaller avalanches are less effective in surrounding the CMB with cold material, and thus a larger fraction of the core flux is taken up in driving active upwellings, and partly because the midmantle region is less hot than in case 1 , due to the smaller thermal boundary layer at $670 \mathrm{~km}$ depth, thus allowing hot plumes from the deep mantle to rise easily through this region.

There has been a tendency to regard plumes, which are postulated to cause hotspots such as Hawaii [e.g., Sleep, 1990; Davies and Richards, 1992], as a distinct, secondary mode of convection, somehow decoupled from the main, platescale flow [Loper and Stacey, 1983; Davies and Richards, 1992]. However, such a mode of flow has never been observed in any self-consistent numerical or laboratory experiment. Upwelling plumes always occur as part of the main, and only, convective system, as we observe in these results. However, there is a problem with obtaining hotspotlike plumes, which must simultaneously satisfy the requirements of being relatively stationary in position and fairly weak, since hotspots are estimated to carry only $6 \%$ of the surface heat flux (excluding the plume head contribution) [Davies and Richards, 1992]. For example, in case 1 the plumes are weak but shortlived and transient, whereas in case 3 the plumes are quite stationary but much stronger than those thought to drive hotspots like Hawaii. It may be that the solution to this problem lies in the use of realistic temperature-dependent viscosity, which we plan to incorporate in future phase change experiments [Tackley, 1993].

On the basis of these observations, three distinct regions of the mantle can be identified: (1) the upper mantle (UM), characterized by linear downwellings and pools of cold material building up above 670, (2) the midmantle (MM), characterized by large cylindrical downwelling plumes and weaker upwelling plumes, and (3) the deep mantle (DM), characterized by pools of avalanched material surrounding the core and a network of interconnected hot ridges which sometimes break through the $\mathrm{MM}$ in the form of plumes to the UM. The UM can be further subdivided into the transition zone (TZ), in which the pools of cold material accumulate, and the shallow mantle (SM), which contains narrow linear downwellings and the upper thermal boundary layer. The next section examines these distinct regions in more detail and discusses their relationship to the surface observables, geoid and dynamic topography.

\section{Surface Observables and Radial Structure}

Plate 3 shows the geoid, dynamic topography, and superadiabatic temperature field for case 1 (with only the 670). The superadiabatic temperature field is shown on four surfaces of constant radius, corresponding to the four distinct regions discussed above: SM, TZ, MM, and DM. This frame corresponds to Plates 1e and 1f. In the SM, the downwelling sheets are clearly visible, separated by broad hot regions. In the $T Z$, broad regions of cold material are visible, caused by pooling of cold material above the 670. At MM depth, the picture is completely different, with a few broad cylindrical downwellings in a generally warm background and a few faint upwelling plumes visible. At DM depth, broad circular cold pools corresponding to the downwellings in $M M$ are seen, with an interconnected system of hot ridges separating them.

The dynamic surface topography is closely related to shallow upper mantle (UM) structure, with the narrow downwelling sheets having a clear surface expression. The geoid, however, resembles a combination of filtered UM and MM structure, with the pattern of upper mantle downwellings visible but negative peaks over the midmantle avalanches. Cold regions are associated with geoid lows, and hot regions are associated with geoid highs.

Plate 4 shows the same plots for case 3 (with a strong $\mathbf{4 0 0}$ added). In this case, there is less pooling of material in the TZ at the intersections of downwellings; rather, a general thickening of the downwellings is observed in this region. At MM depth, there are more avalanches visible, typically smaller than in case 1, and including at least one which is elongated. The surface topography and geoid display the same characteristics as in case 1 , with the geoid even more strongly reflecting upper mantle structure and the position of MM avalanches barely visible.

As shown in various studies [Ricard et al., 1984; Richards and Hager, 1984; Hager and Clayton, 1989], the net geoid resulting from, for example, a cold temperature anomaly (i.e., a positive mass anomaly) is the small difference of two large terms, the geoid high associated with the anomaly itself and the geoid low associated with the dynamically induced negative topography at the surface and $\mathrm{CMB}$. The sign and amplitude of the observed geoid are highly sensitive to the exact details of the radial viscosity profile and any chemical stratification. For a simple constant viscosity profile and chemically homogeneous mantle, the contribution from surface topography is always dominant, and thus the net geoid associated with a cold temperature anomaly is a geoid low, as observed in our model.

These different contributions can be combined to obtain kernels giving the observed geoid for sheet mass anomalies of different spherical harmonic degrees at different depths. These kernels go to zero at the boundaries due to perfect isostatic compensation of mass anomalies at chemical boundaries. For a homogeneous, constant-viscosity mantle, the long-wave- 


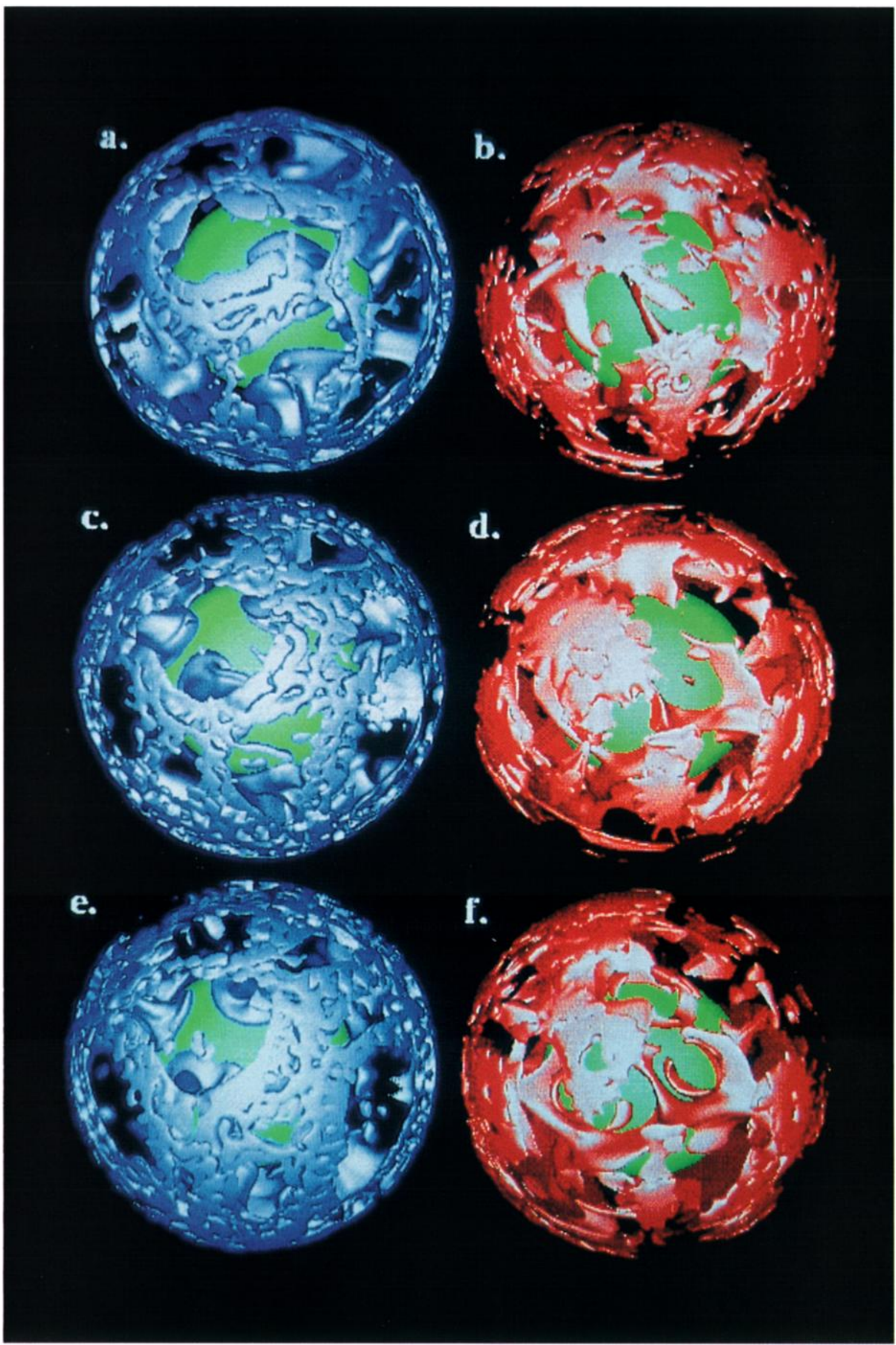

Plate 2. As Plate 1, but for case 3 (both phase changes), again at intervals of around $500 \mathrm{~m} . \mathrm{y}$. 

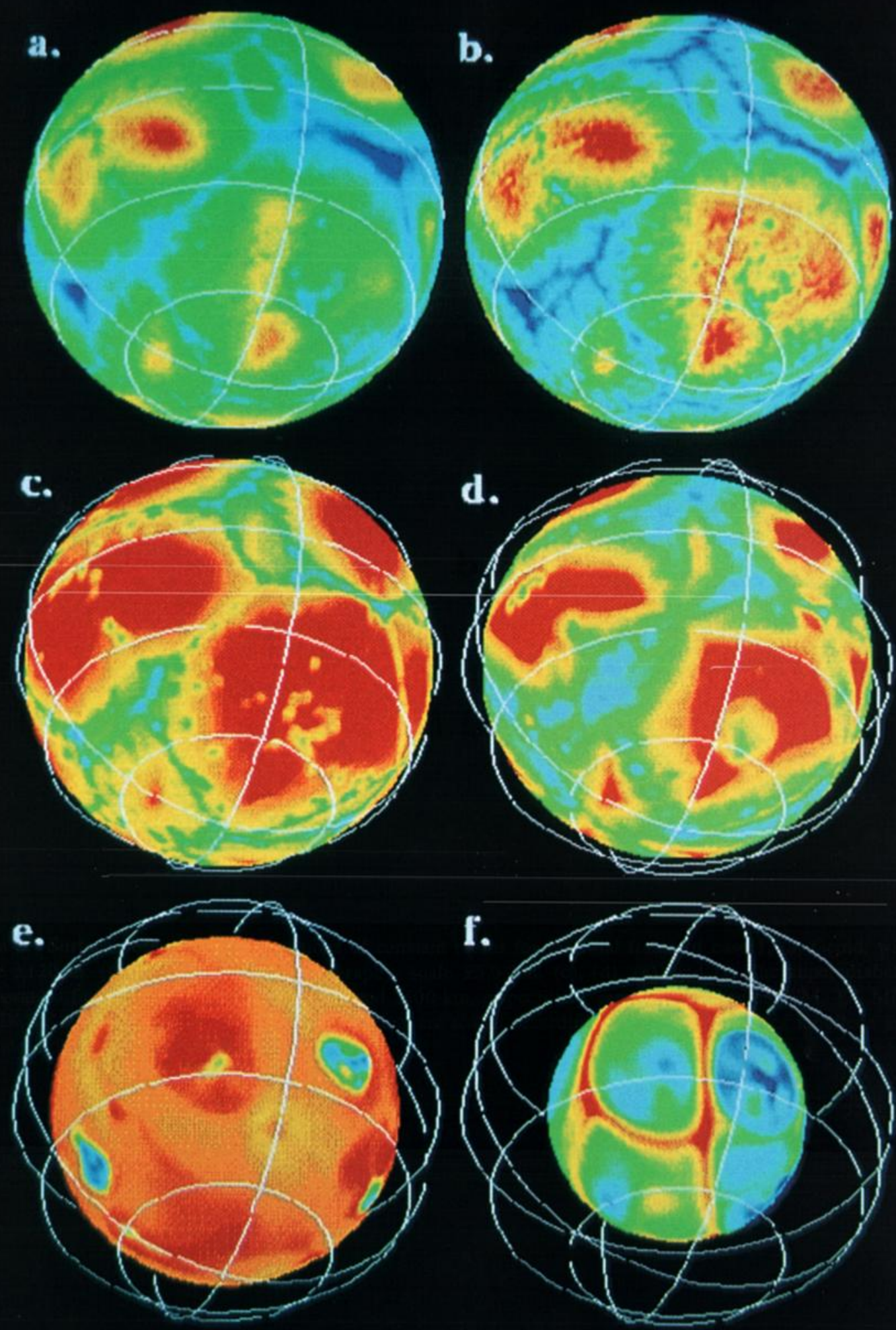

Plate 3. Surface observables, and sections of constant radius for the final frame of case 1. (a) Geoid, with scale $\pm 120 \mathrm{~m}$. (b) Dynamic surface topography, scale $\pm 5.0 \mathrm{~km}$. (c), (d), (e), and (f) The superadiabatic temperature field at depths $200,550,1200$, and $2600 \mathrm{~km}$, respectively, representing regions SM, TZ, MM, and DM. Scale ranges from -1050 to $+350 \mathrm{~K}$. Plots are scaled according to the radius; lines of longitude and latitude indicate the position of the surface. 

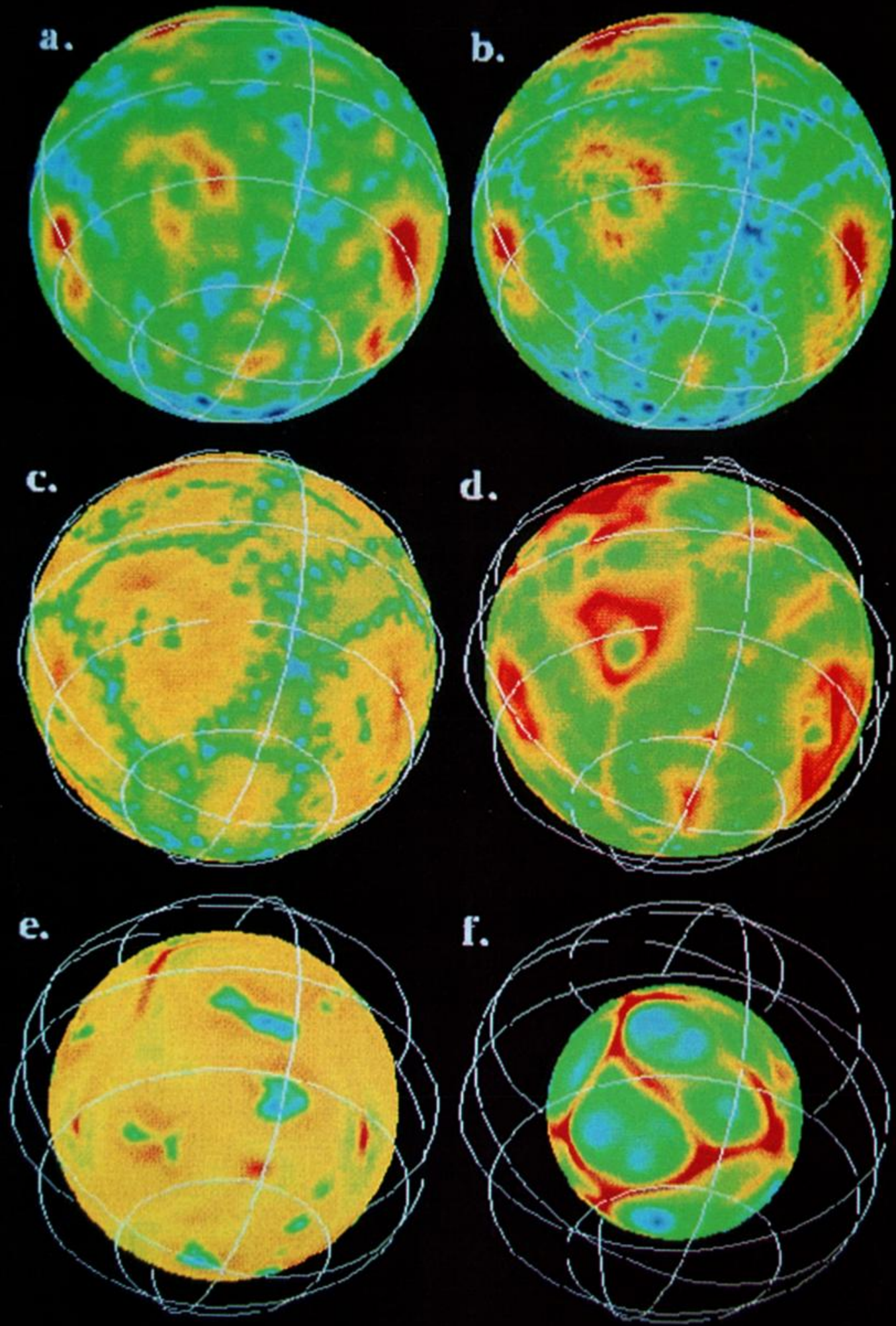

Plate 4. As Plate 3, but for case 3 . Geoid ranges $\pm 110 \mathrm{~m}$, topography ranges $\pm 5.0 \mathrm{~km}$, and superadiabatic temperature ranges from -950 to $+300 \mathrm{~K}$. 
length kernels peak deep in the interior, with $\ell=2$ peaking at around midmantle depth, $\ell=8$ peaking in the transition zone, and shorter wavelengths peaking at progressively shallower depths [Hager and Richards, 1989; Hager and Clayton, 1989]. This explains why the geoid signal observed in our models, which is dominated by long wavelengths, is closely related to structure in the $\mathrm{TZ}$ and $\mathrm{MM}$.

The downward deflection of the phase boundary at $670 \mathrm{~km}$ depth caused by a cold temperature anomaly (resulting in a positive mass anomaly) at that depth results in a negative mass anomaly. Thus the geoid contributions of these opposite anomalies partially cancel, reducing the geoid contribution from transition zone heterogeneity. In contrast, deflection of the 400 results in a geoid contribution of the same sense to temperature anomalies in that region, enhancing the geoid contribution from the mid upper mantle. This partly accounts for the differences between case 1 , in which geoid resembles a combination of UM and MM structure, and case 3, in which the UM completely dominates the geoid signal.

The major downwellings (slabs) of Earth are associated with geoid highs rather than geoid lows, as in our model. This discrepancy could be corrected by a large viscosity jump at $670 \mathrm{~km}$ depth [Hager and Clayton, 1989] which is a common feature of mantle viscosity models derived using geoid data [e.g., Davies and Richards, 1992; King and Masters, 1992; Forte et al., 1993].

\section{TIme Dependence}

Plate 5 shows the geoid, TZ and MM structure, and crosssections for case 1 at three times corresponding to the same times and viewing angle as in Plate 1 . The radial dependence of the flow structure is clearly visible in the right plots (Plates $5 \mathrm{~d}, 5 \mathrm{~h}$, and 5l), which show the superadiabatic temperature field in constant-longitude cross sections. The upper mantle is clearly heterogeneous on long wavelengths, containing some broad (up to $-10,000 \mathrm{~km}$ ) regions of anomalously cold or hot material, as well as regions with the classical small aspect ratio convection cells [Christensen and Yuen, 1988; Busse, 1989]. Pooling of cold material can be seen in the TZ. The MM and DM can be clearly distinguished, with DM occupying the lowermost $\sim 800 \mathrm{~km}$ of the mantle.

Comparing the geoid with the TZ and MM for the three times, it appears that the geoid closely resembles upper mantle structure. Avalanche conduits in the MM are associated with distinct geoid lows that are of only slightly higher amplitude than the signature of the upper mantle structure.

The same plots for case 3 (Plate 6) reveal a similar behavior. In the MM region, distinct hot upwelling structures can be identified more clearly than in case 1 . However, in this case, avalanche conduits have a weaker surface expression, which is partly because the midmantle is colder, thus reducing the temperature anomaly associated with avalanche conduits,

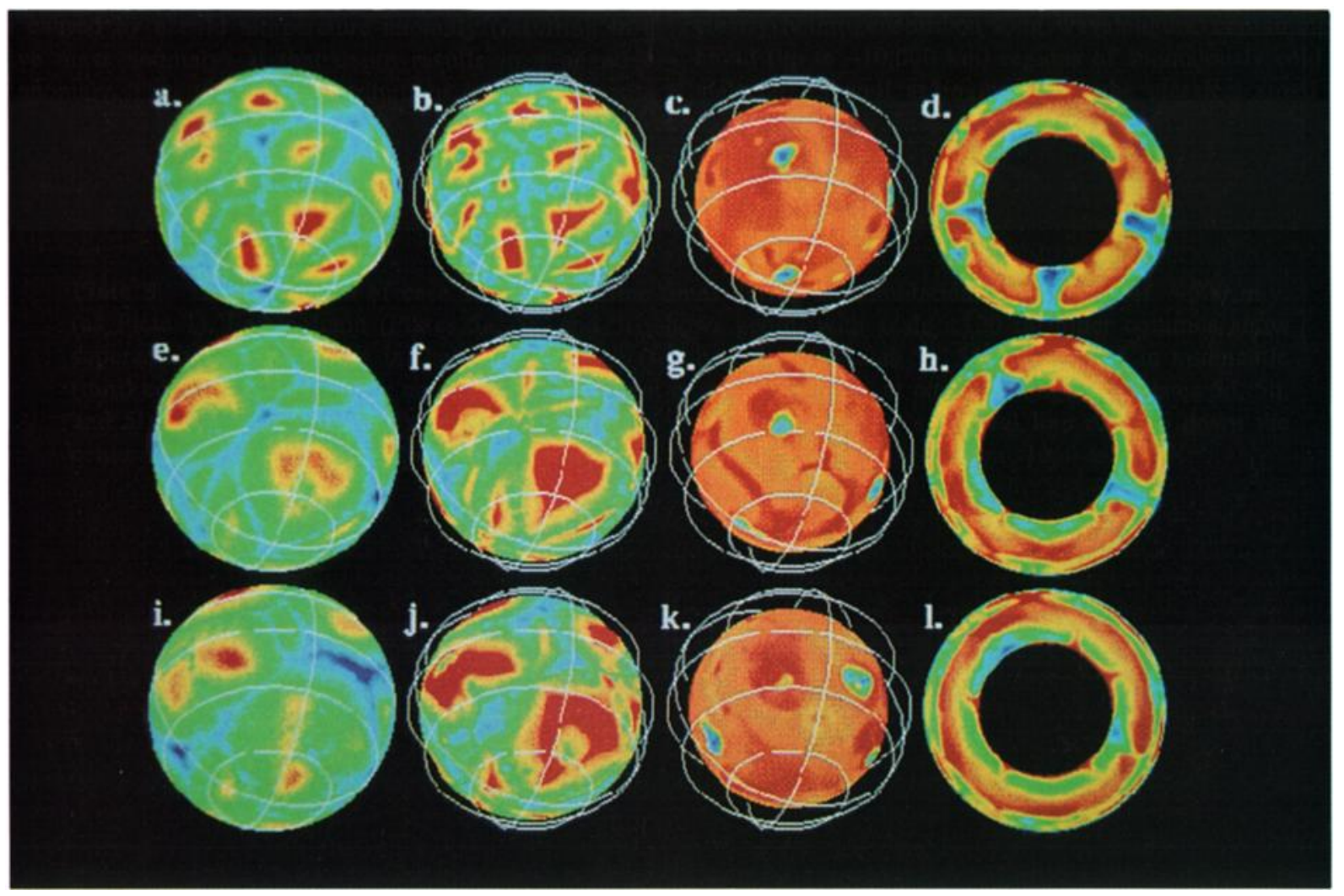

Plate 5. Time evolution of case 1 , showing three times during the simulation, at intervals of $-500 \mathrm{~m} . \mathrm{y}$. (as Plate 1). Left column (Plates 5a, 5e, and 5i) shows geoid, with scale $\pm 120 \mathrm{~m}$, other columns show superadiabatic temperature in the transition zone (second column, Plates $5 \mathrm{~b}, 5 \mathrm{f}$, and $5 \mathrm{j}$ ) and in midmantle (third column, Plates $5 \mathrm{c}, 5 \mathrm{~g}$, and $5 \mathrm{k}$ ) and in vertical cross section (i.e., at constant longitude, Plates 5d, 5h, and 51 ), with the right-hand side of the cross sections corresponding to a vertical line running down the center of the constant-radius sections. Superadiabatic temperature scale ranges from -1050 to $350 \mathrm{~K}$. 


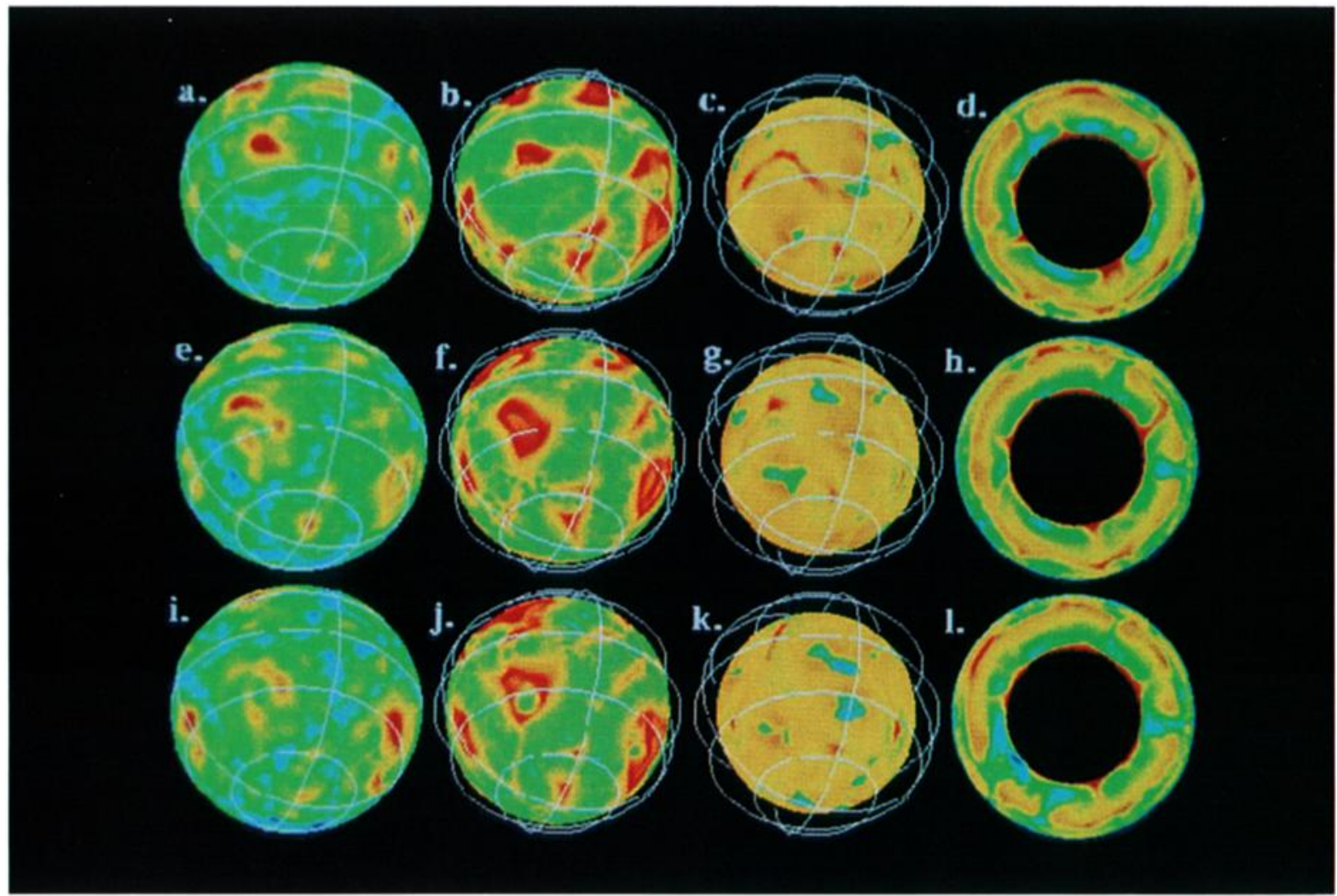

Plate 6. As Plate 3, for case 3. Geoid ranges $\pm 110 \mathrm{~m}$, and superadiabatic temperature ranges from -950 to $+300 \mathrm{~K}$.

and partly because the contribution from 400 deflection reinforces the upper-mantle signature, as discussed earlier.

\section{Spherically Averaged Temperature}

The horizontally averaged $(\ell=0)$ superadiabatic temperature fields for all cases are shown in Figure 1 . Due to slightly different reference adiabats in different cases, these have been aligned at the surface and CMB. In general, both the upper and lower mantles are strongly subadiabatic. There is a strong boundary layer at $670 \mathrm{~km}$, having a temperature change of several hundred degrees over a vertical extent of $-250 \mathrm{~km}$, in addition to the usual boundary layers at the surface and $\mathrm{CMB}$. Small kinks, of vertical extent $\sim 30 \mathrm{~km}$, are observed at 670 $\mathrm{km}$ and $400 \mathrm{~km}$, due to the release or absorption of latent heat by material advected across the phase change. This effect was previously noted by Christensen and Yuen [1985]. In regions of high vertical mass flux (i.e., high local Peclet number) at $670 \mathrm{~km}$ depth, the release or absorption of latent heat is dominant and local conductive heat flux is negative (i.e., downward). However, in regions where cold material is pooling above the 670 and thus the radial velocity through the 670 is very low, the resulting thermal boundary layer results in strong upward conduction of heat. When spherically averaged, these effects nearly cancel in a very localized region around the 670 , so that the net thermal gradient at $670 \mathrm{~km}$ depth is low, and thus the net thermal conduction across the 670 is close to zero, and most of the heat flux across $670 \mathrm{~km}$ is advected. The subadiabatic upper mantle and the thermal boundary layer across the 670 result in a local temperature minimum in the transition zone [Weinstein, 1993], which, if temperature-dependent viscosity were included, would result in higher viscosity in this region.
Comparing cases 1,2 , and 3 , it is clear that the magnitude of the thermal boundary layer across the 670 diminishes as $P_{400}$ increases due to an increase in either Clapeyron slope or density change. Thus it seems reasonable to conclude that the amount of layering decreases due to the presence of the 400 . This decrease in the temperature change across the 670 is associated mainly with a reduction of the temperature in the $M M$,

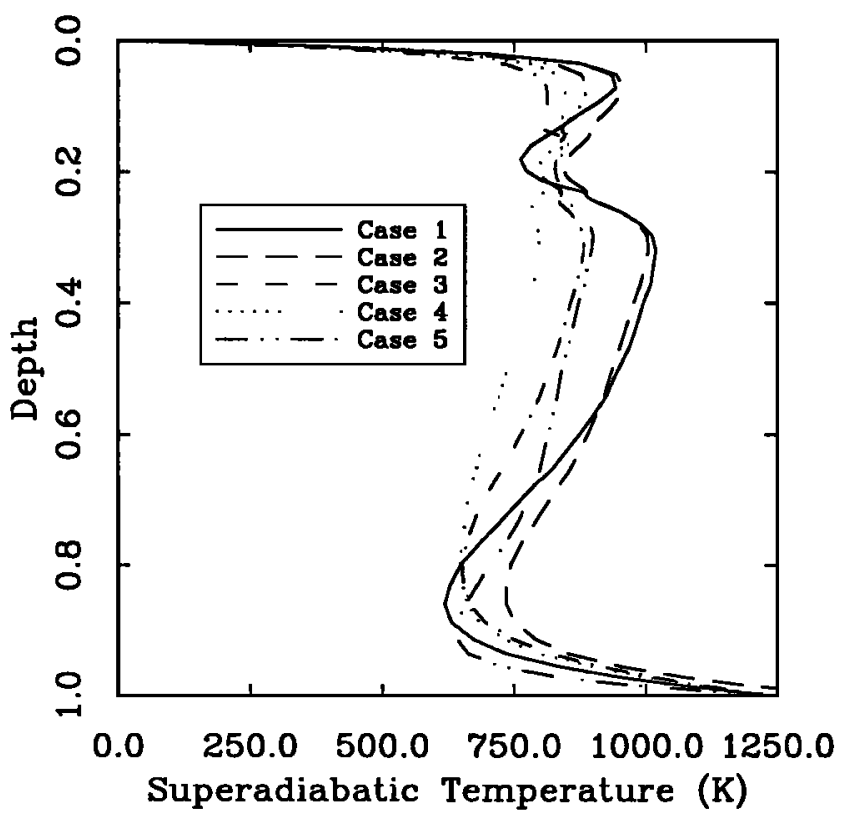

Figure 1. Radial profile of spherically averaged $(\ell=0)$ superadiabatic temperature for cases $1-5$. Profiles are aligned by boundary conditions. 
resulting in easier penetration of upwelling hot plumes from the DM. Case 4, which has a weaker 670 Clapeyron slope of -3 MPa K$~^{-1}$ but otherwise the same parameters as case 2, displays virtually no boundary layer at $670 \mathrm{~km}$ depth, suggesting that the strength of the 670 exerts a much greater influence on stratification than the strength of the 400 .

In the DM, where avalanches spread out into pools of cold material surrounding the core, the temperature is strongly depressed from an adiabat extrapolated from the upper mantle. This effect is greatly enhanced by the spherical geometry, because the surface area of the core is $-35 \%$ of the surface area of the $670-\mathrm{km}$ interface. The departure of the geotherm from an adiabat is of similar magnitude to the rms temperature variations in the interior but a factor of $\mathbf{- 5}$ lower than the peak-topeak temperature variations at a particular depth. A subadiabatic interior temperature gradient is expected in a mantle beated substantially from within [Schubert, 1992; Parmentier et al., 1994]. However, the avalanches strongly increase this subadiabaticity. This region of depressed temperature in the deep mantle is important in the interpretation of seismic tomography, as discussed later.

In case 5 , in which the viscosity is constant with depth, the DM region has a smaller vertical extent, as can be seen by examination of the temperature profiles in Figure 1. The thermal gradient at the CMB is steeper, resulting in a higher core heat flux. These effects arise because cold avalanched material is not slowed down by a viscosity increase in the deep mantle, as it is in the other cases.

\section{Radial Mass Flux}

A useful indicator of the degree of flow stratification is the radial mass flux diagnostic [Peltier and Solheim, 1992], defined as the spherically averaged absolute radial mass flux, normalized so that the integral over nondimensional depth is unity:

$$
f(r)=\frac{\left\langle\rho\left|u_{r}\right|>\right.}{y_{\Delta r} \int<\rho\left|u_{r}\right|>d r}
$$

where $\langle p l u$,$\rangle is the absolute value of the mass flux averaged$ over a surface of constant radius, the integral in the denominator is performed from the CMB to surface, and $\Delta r$ is $r_{\text {surface }}$ $r_{\mathrm{cmb}}$.

The radial mass flux diagnostic for the various cases, averaged over several thousand time steps, is shown in Figure 2. The phase change is observed to have a marked inhibitive effect on the flow, as indicated by the minimum in the radial mass flux at $670 \mathrm{~km}$. Comparison of cases 1-3 indicates the effect of the 400 on the propensity to layering. The most pronounced minimum at $670 \mathrm{~km}$ is shown by case 1 , which has no phase change at $400 \mathrm{~km}$. Cases 2 and 3 , with increasingly stronger phase changes at $400 \mathrm{~km}\left(P_{400}\right.$ is 0.055 and 0.135 , respectively), display progressively less pronounced minima, indicating that increasing the strength of the 400 decreases the degree of layering. This was also indicated by examination of the spherically averaged temperature profile (previous section). Solheim and Peltier [1994] reach the same conclusion based on 2-D spherical axisymmetric modeling, but Steinbach and Yuen [1992] and Zhao et al. [1992] find that adding the 400 decreases the mass flux across $670 \mathrm{~km}$ in their 2-D Cartesian experiments. This

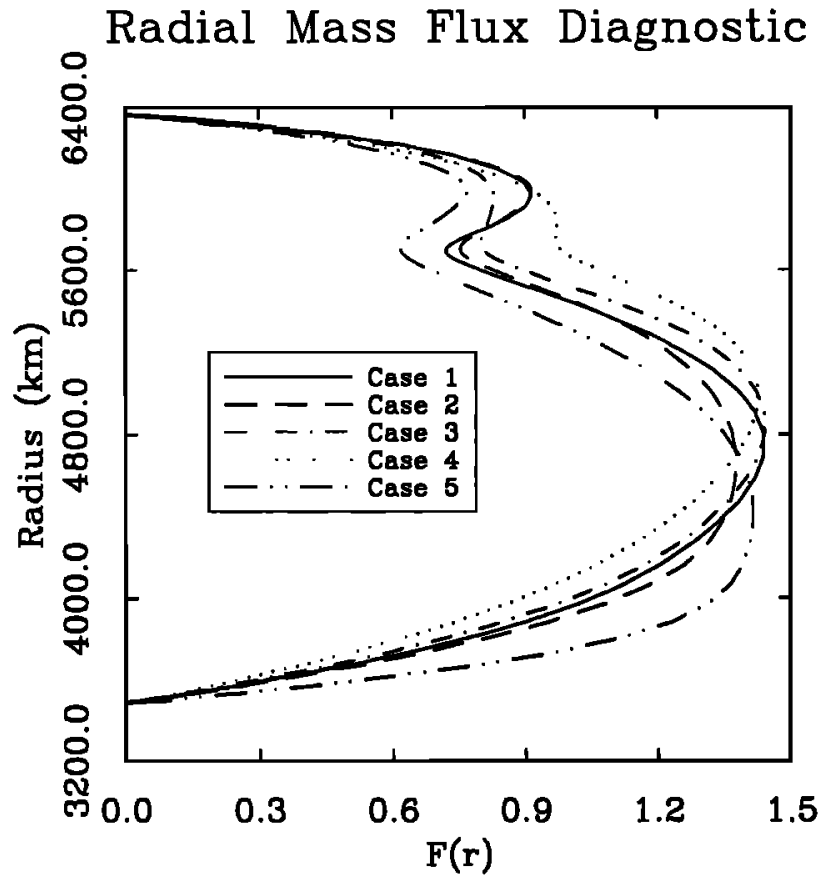

Figure 2. Radial mass flux diagnostic for cases 1-5. See text for definition.

difference could be related to the depths at which the phase changes are placed or the difference between Cartesian and spherical geometries. It does not seem to be related to Rayleigh number or dimensionality since our experiments are almost an order of magnitude lower in Rayleigh number and have an additional dimension, compared with those of Solheim and Peltier [1994], but we reach the same conclusion.

For case 4, which has a weaker 670 Clapeyron slope of -3 $\mathrm{MPa} \mathrm{K} \mathrm{K}^{-1}$ and the same 400 strength as case 2 , the radial mass flux diagnostic displays no minimum but just a change in slope at $670 \mathrm{~km}$ depth. Thus the degree of layering is highly sensitive to the strength of the 670 . From a comparison of cases 1 to 4, it is evident that the degree of layering is much more sensitive to the strength of the phase change at $670 \mathrm{~km}$ depth than the strength of the phase change at $400 \mathrm{~km}$ depth. The constant viscosity case (case 5) displays a similar amount of layering as case 2 but has greater mass flux towards the base of the mantle, presumably due to the lower viscosity there.

Examination of the radial mass flux for individual spherical harmonic degrees (i.e., the spherical harmonic coefficients of $p u_{r}$ on surfaces of constant radius) for the most stratified case (case 1, Figure 3) indicates that long wavelengths of flow, such as $\ell=2$ and $\ell=5$, are virtually unaffected by the phase change, whereas short wavelengths are increasingly inhibited, with a noticeable effect at $\ell=10$; and flow for spherical harmonic degrees above around 40 is effectively confined to the upper mantle. At the wavelengths employed in the current generation of global seismic tomographic models, the flow appears essentially unlayered, compatible with the long-wavelength flow pattern in Earth calculated by Phipps Morgan and Shearer [1993], which takes into account density anomalies derived both from global seismic tomography and from the observed deflection of the 670 [Shearer and Masters, 1992]. A qualitative explanation of this phenomenon is as follows: At a particular point, flow with a given horizontal wavelength is sensitive to density anomalies vertically averaged over a 


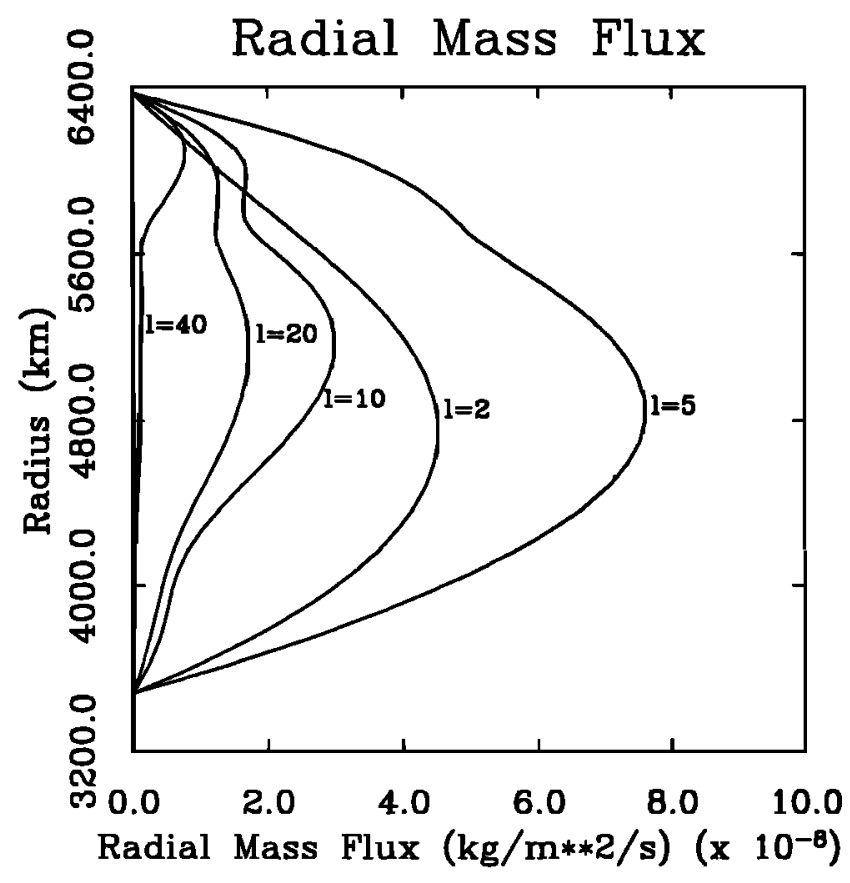

Figure 3. Radial mass flux for case 1, at individual spherical harmonic degrees $(\ell)$ of $2,5,10,20$, and 40 , as indicated.

length scale similar to this horizontal wavelength. For short wavelengths, flow through the 670 is sensitive only to density anomalies in the immediate vicinity of the 670 and thus is strongly influenced by deflection of this interface. At the longest wavelengths, however, flow across the 670 is influenced by density anomalies vertically averaged through the entire mantle, and thus a much larger deflection of the 670 would be needed to balance this integrated buoyancy and thereby prevent flow penetration. Deflection of a downwelling by the 670 is associated with short wavelengths of flow; avalanches are associated with long wavelengths of flow. The calculation that the long-wavelength flow field in Earth penetrates the 670 is still compatible with slabs being deflected when they first encounter the phase transition.

From the radial mass flux across the interface at $670 \mathrm{~km}$ depth it is possible to calculate a "mixing time", defined as the time required for a mass equal to the mass of the mantle to pass through $670 \mathrm{~km}$. For cases $1-5$ this time is $4.5,4.3,4.1,2.3$, and 3.7 b.y. respectively. This further quantifies the conclusions discussed above: the effect of the $\mathbf{4 0 0}$ is to moderately decrease the propensity to layering, while the effect of slightly weakening the 670 is to greatly decrease the degree of layering. To scale these mixing times to Earth's Rayleigh number (Ra), it is likely that the mass flux (of downwelling cold material) scales roughly as the heat flux, suggesting a $R a^{1 / 3}$ scaling [Turcotte and Schubert, 1982] which would reduce the mixing time to $-2.1,2.0,1.9,1.1$, and 1.7 b.y., respectively, for an order of magnitude increase in $R a$. This is less than half the age of Earth, but the increased effect of the phase change at higher $R a$ [Christensen and Yuen, 1985; Yuen et al., 1994] may increase this time.

\section{Globally Averaged Time Dependence}

The time dependence of radial mass flux through the phase changes and total heat flow through the CMB and surface are shown for the last billion years of case 3 in Figure 4. There is some time dependence of the flow through the phase changes at 400 and $670 \mathrm{~km}$ depth, with the mass flux diagnostic at 670 $\mathrm{km}$ depth $\left(F_{670}\right)$ varying between 0.75 and 0.85 during this period. $F_{400}$ and $F_{670}$ display similar variations, with $F_{400}$ generally higher than $F_{670}$, except during one brief period toward the end of the simulation. The surface and CMB heat fluxes display very weak time dependence, in contrast to the two-dimensional calculation of Weinstein [1993], in which they show a sharp peak for each individual avalanche.

These plots show that the time dependence of globally averaged quantities is much weaker than that observed in spherical axisymmetric [Machetel and Weber, 1991; Peltier and Solheim, 1992] or 2-D Cartesian [Weinstein, 1993] geometries, because due to the large effective aspect ratio of the sphere, several avalanches are occurring at any given time, and thus the convection is never strongly layered on a global scale. As a result, globally averaged diagnostics such as $\mathbf{F}_{670}$, surface beat flux, mean temperature, etc., are not greatly affected by an individual avalanche.

\section{a. Radial Mass Flux}
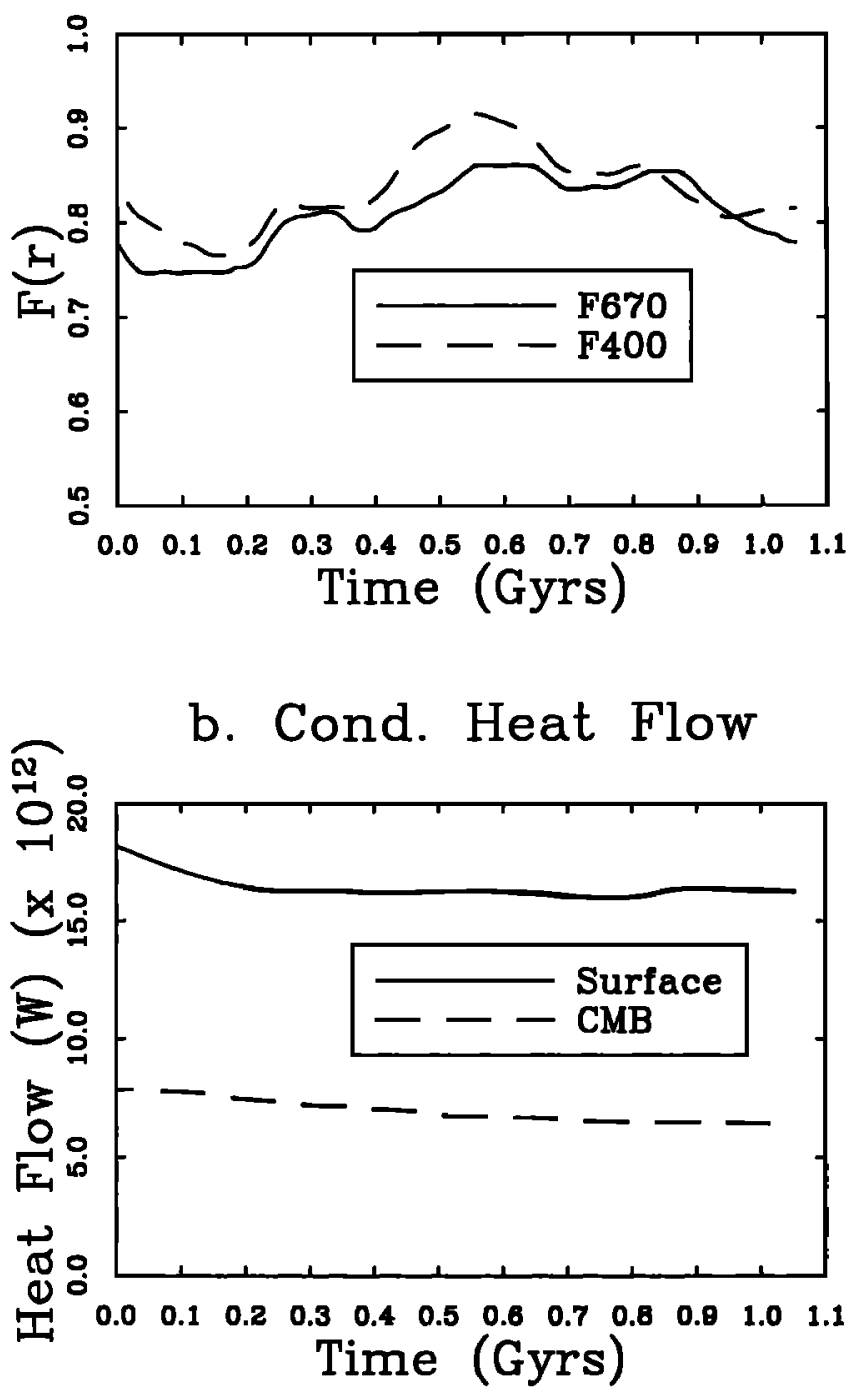

Flgure 4. Time dependence of mass and heat fluxes for the last billion years of case 3. (a) Radial mass flux diagnosic at 400 and $670 \mathrm{~km}$ depth. (b) Conductive heat flux at surface, 400 $\mathrm{km}$ depth, $670 \mathrm{~km}$ depth, and CMB. 
It is possible that our avalanches would be more abrupt and violent if we could decrease the viscosity (thus increasing the Rayleigh number) to Earth-like values, as observed in twodimensional models [Weinstein, 1993; Solheim and Peltier, 1994]. However, we believe that with an Earth-like viscosity, avalanches would still overlap in time and the global time dependence would be intrinsically weaker in full three-dimensional spherical geometry than in spherical axisymmetric or two-dimensional Cartesian geometries. Four arguments support this view: (1) Cylindrical downflows penetrate the endothermic phase change more easily than linear downflows and thus result in smaller, less vigorous and more frequent avalanches. This is indicated by our numerical results, and by the analytical model of Bercovici et al. [1993]. (2) Cylindrical avalanches last for longer, thus increasing the probability of temporal overlap with other avalanches. This is because they take longer to exhaust their pool of feeding material than linear avalanches, due to the radially convergent influx of material towards the conduit. (3) The synchronicity observed in two dimensions, whereby an avalanche in one place induces a simultaneous avalanche in another place, is less likely to occur in three dimensions. This is because the velocity field associated with 3-D cylindrical downflows decreases with distance much more rapidly than that for a linear downflow (1/distance rather than $1 / \log$ (distance)), and thus cylindrical avalanches will have a much smaller influence on their distant surroundings. (4) If avalanches are essentially unsynchronized, there is a much higher probability of temporal overlap in a 3-D sphere than in an axisymmetric sphere, because due to simple geometry, it is possible to fit many simultaneous cylindrical avalanches into a 3-D sphere, but only about three linear avalanches in a 2-D axisymmetric sphere.

\section{Comparisons With Earth}

\section{Lateral Heterogenelty}

The effect of the phase changes, particularly the endothermic transition at $670 \mathrm{~km}$ depth, on the spectrum of density anomalies at different radii is pronounced. Previous work has focused on the depth dependence of total power, which shows a peak at $670 \mathrm{~km}$ [Peltier and Solheim, 1992; Tackley et al., 1993], and the vertically averaged lateral spectrum, which for case 1 shows some similarity with the global seismic tomographic model of Inoue et al. [1990] [Tackley et al., 1993]. Here we investigate the spectral characteristics further by considering spectral heterogeneity maps (i.e., the long-wavelength spherical harmonic spectrum as a function of radius) for cases 1 and 4 , which are the most stratified and least stratified cases, respectively, and the seismic model SH12/WM13 of Su et al. [1992b]. Such comparisons between convection models and seismic models are made complicated by the problem of an uncertain radially dependent coefficient to scale between entropy or temperature and seismic velocity. In particular, this coefficient may be much higher in the transition zone than in the surrounding layers (suggested by the inversion of Forte et al. [1993] and supported by the thermodynamic analysis of Karato [1993]), which would suppress transition zone heterogeneity in the seismic models. The problem of this uncertain radially dependent coefficient can be eliminated by normalizing the spectrum at each radius by the total rms amplitude at that radius. Thus we present normalized spectra in addition to unnormalized spectra which still retain information about radial variation of total rms amplitude.

In the spectral heterogeneity map (SHM) for case 1 (Figures $5 \mathrm{a}$ and $5 \mathrm{~b}$ ), the three distinct regions, previously identified from the spatial images of the temperature field, are clearly visible from their spectral characteristics. The upper mantle (UM) is characterized by a high-amplitude "red" (i.e., longwavelength, peaking at $\ell<8$ ) spectrum, although since it also contains narrow features, there is still significant amplitude at much higher degrees, as may be true in Earth [Gudmundsson et al., 1990; Davies et al., 1992]. The midmantle (MM), comprising the upper $-1000 \mathrm{~km}$ of the lower mantle, is characterized by a low-amplitude, broad spectrum, with a wide peak at $\ell=5-20$, and the deep mantle (DM) is again characterized by a high-amplitude red spectrum (peak $\ell<6$ ). These can be understood qualitatively by the following argument: Jarvis and Peltier [1986] showed that for simple convection cells, boundary layers are characterized by a highamplitude, long-wavelength spectrum, with the interior of the convective region having a low amplitude, broad spectrum. Thus the UM and DM display the boundary layer signature, while the MM corresponds to the interior of the convective region. The entire UM has a boundary layer signature due to the pooling of cold material above the 670 , which also creates an additional boundary layer at $670 \mathrm{~km}$ depth, identified by a peak in the total power [Tackley et al., 1993; Peltier and Solheim, 1992]. Avalanched cold material pooling above the $\mathrm{CMB}$ gives the DM its long-wavelength signature. The height of this region is influenced by the magnitude of the viscosity increase with depth, as shown earlier by consideration of spherically-averaged temperature profiles.

Figures 5c and 5d show the SHM for case 4, the case with the least stratification, as discussed earlier. Even with the very small degree of layering, the three main regions are again visible, though less clearly, indicating that long-wavelength averaged characteristics of the thermal field are very sensitive to even small degrees of flow stratification. The upper boundary layer is much stronger than the $670-\mathrm{km}$ boundary layer (Figure 5c), whereas they are of comparable amplitude in case 1 (Figure 5a). In addition, the spectral peak has shifted to shorter wavelengths and broadened in this case.

The raw seismic model (Figure 5e) does not display much rms beterogeneity in the transition zone or a peak in total amplitude at $670 \mathrm{~km}$ depth. This is also true of other seismic models we have examined [Tanimoto, 1990b; Inoue et al., 1990; Masters et al., 1992]. However, when normalized, a more coherent upper mantle signature emerges, with a change in the spectral characteristics at around $670 \mathrm{~km}$ depth, marking the start of the MM. The DM is visible in both raw and normalized spectra and extends over a somewhat greater depth range than in the convection models.

Thermal fields from the convection models lack the dominant $\ell=2$ component found in some seismic models [Nakanishi and Anderson, 1983; Tanimoto, 1990a,b; Su and Dziewonski, 1991, 1992]; possible reasons for this are discussed later. The asymptotic behavior of power with spherical harmonic degree varies with depth but goes approximately as $\ell^{-2}$.

\section{Geold Spectra}

Figure 6 shows the amplitude spectra of the geoid (i.e., the total geoid amplitude at each spherical harmonic degree, 

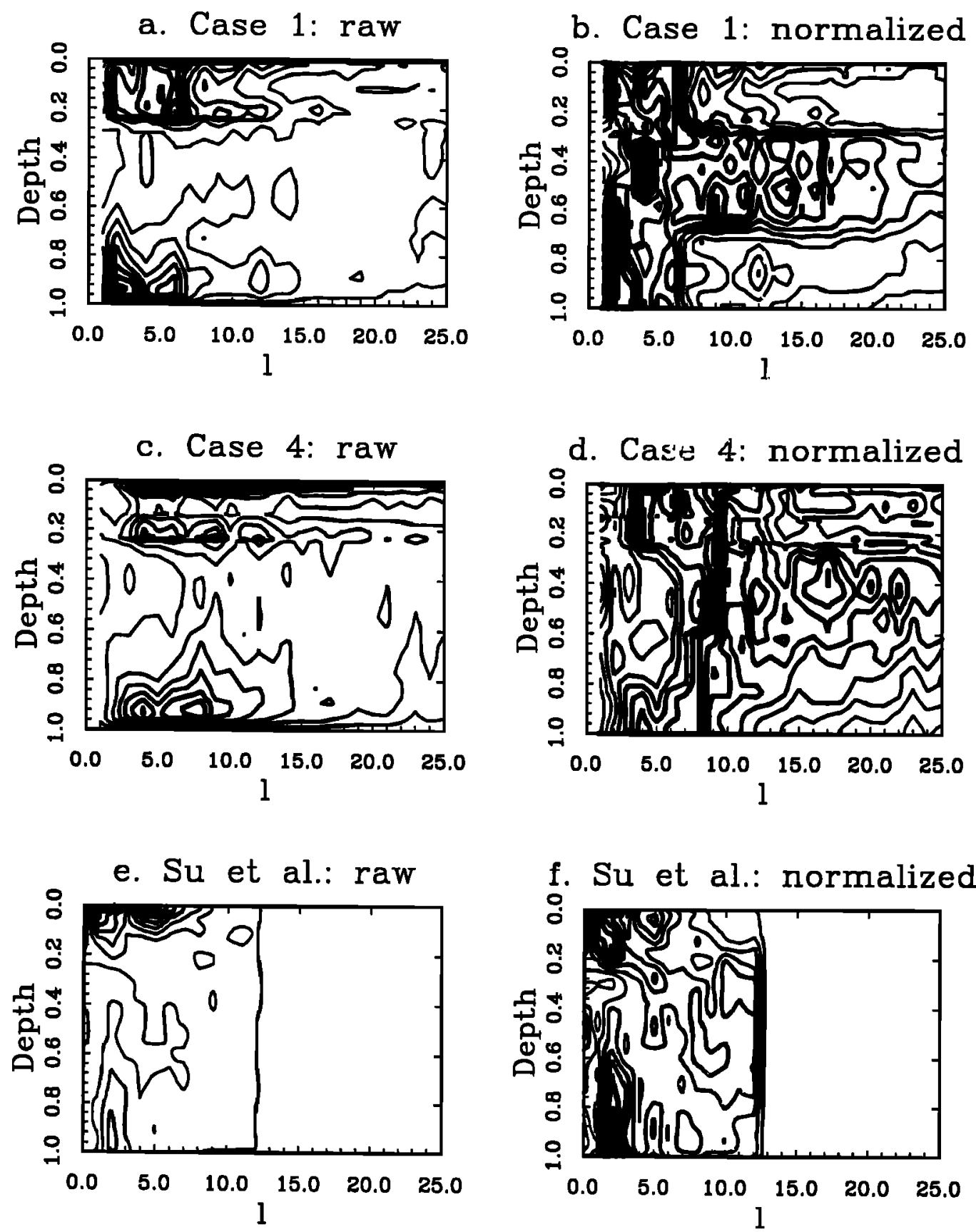

Figure 5. Spectral heterogeneity maps (SHM) for cases 1 and 4 and the seismic model SH12/WM13 of $S u$ et al. [1992b]. Plotted are contours of rms spherical harmonic amplitude as a function of (normalized) depth. In the left column are the entropy perturbations (for simulations) and seismic velocity anomalies (for seismic model). The plots in the right column have been normalized by the total rms amplitude at each radius. Thickness of contour line is proportional to amplitude, with $\mathbf{1 0}$ contour levels scaled to maximum value in each plot.

summed over order) for four convection models, compared to observations of Earth [Rapp and Pavlis, 1990] and Venus (A.S. Konopliv, personal communication, 1993). The geoid is normalized to the radius of the planet, which is equivalent to normalizing gravitational potential to $G M / R$, where $G$ is the gravitational constant, $M$ is the planetary mass, and $R$ is the planetary radius. The convection models are case 1 (Figure 6a), case 2 (Figure $6 \mathrm{~b}$ ), an $85 \%$ internally heated case with no phase change, reported by Tackley et al. [1993] (Figure 6c), and an unpublished case with no internal heating, no phase change, constant viscosity, and a Rayleigh number of $3 \times 10^{6}$ (Figure 12d).

There are two main characteristics to compare: the slope $(\partial \ln A / \partial \ln \ell)$ and the absolute amplitude. All simulations and data display roughly the same slope $(\partial \ln A / \partial \ln \ell \equiv-1.5)$ and absolute amplitudes, except for the internally beated, no phase change case (Figure 6c), which has a plateau for low degrees up to -20-30. The absolute amplitudes of geoid coefficients for the simulations with phase changes are broadly comparable to those of Earth and Venus and to the basally heated case with no 

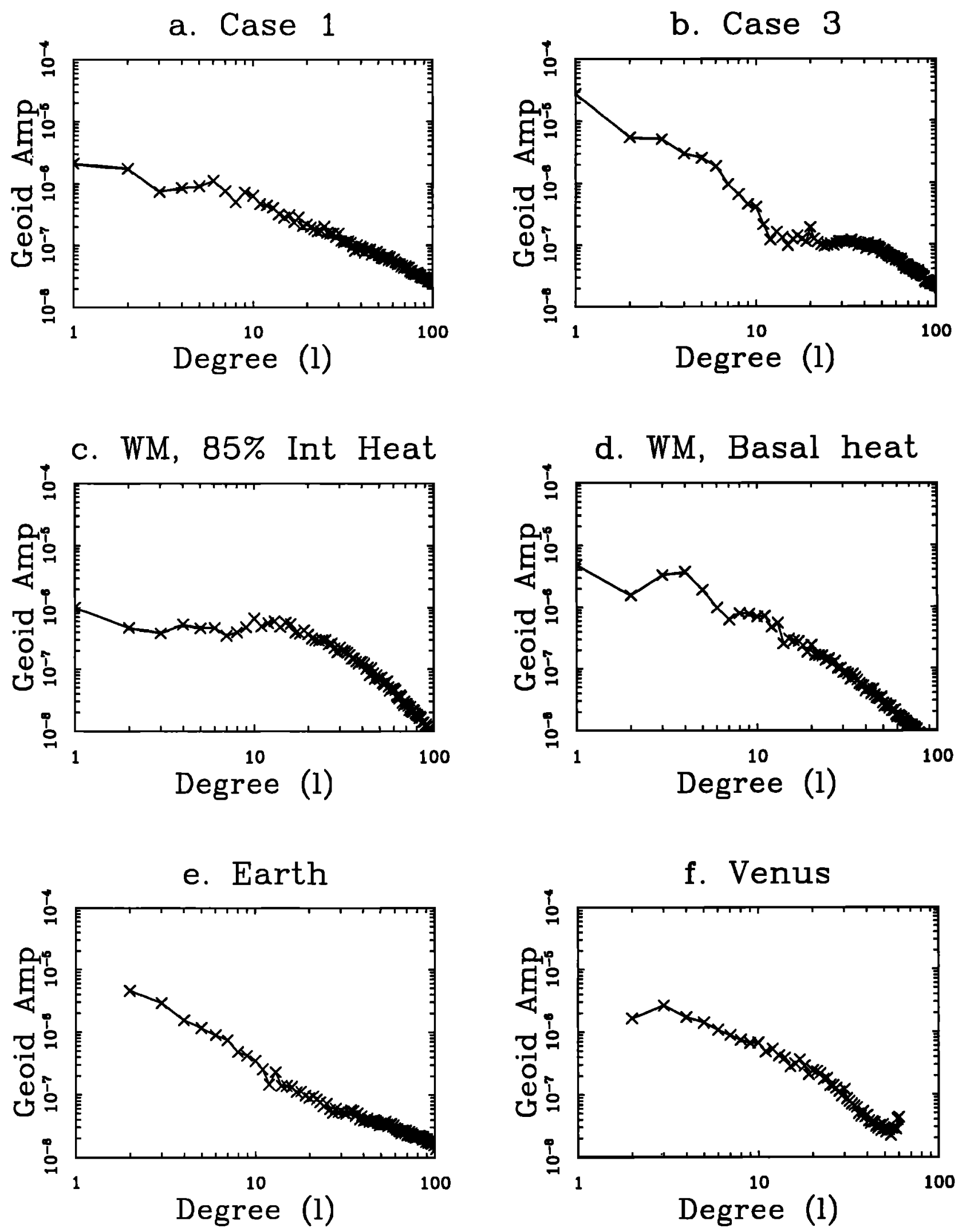

Figure 6. Geoid amplitude spectra, normalized to planetary radius, for four convection models, compared to Earth and Venus. (a) Case 1. (b) Case 3. (c) Whole mantle model with $85 \%$ internal heating. (d) Whole mantle model with completely basal heating. (e) Earth. (f) Venus. 
phase change, although there are differences in detailed degreeby-degree comparisons.

This is somewhat surprising, since (1) the geoid kernels (discussed earlier) for low degrees peak at midmantle $(\ell=2)$ to transition zone $(\ell=8)$ depths, falling to zero at the surface and CMB [Hager and Clayton, 1989], and (2) the phase change introduces high-amplitude, long-wavelength heterogeneity in the transition zone, where these geoid kernels are most sensitive. Thus one would expect a much larger geoid, an argument that has been used in the past to argue against any significant layering [Davies and Richards, 1992, and references therein]. The lack of any significant signal from the strong transition zone heterogeneity is due to the near canceling of its contribution by an opposite and nearly equal contribution from deflection of the $670-\mathrm{km}$ interface. In this dynamically self-consistent system, cold material accumulates in the transition zone while its negative buoyancy is balanced by positive buoyancy from downward deflection of the phase boundary. A balancing of mass anomalies close together results in a near canceling of geoid contributions. The cases with no phase changes have their long-wavelength power concentrated in the surface and CMB thermal boundary layers [Jarvis and Peltier, 1986], where the long-wavelength geoid kernels are close to zero.

The $\ell=1$ geoid is usually set to zero by taking the origin of coordinates to be the center of mass. However, in these simulations the coordinate origin is fixed, and it is interesting to note that in case 1 and the two whole mantle cases, the $\ell=1$ coefficient was the largest, indicating offsets (up to $-24 \mathrm{~m}$ ) of the fixed coordinate origin (the center of figure) from the instantaneous center of mass. This is much smaller than the observed offsets of $280 \mathrm{~m}$ for Venus (D.L. Bindschadler et al., Venus's center of figure-Center of mass offset, submitted to Icarus) and $2.1 \mathrm{~km}$ for Earth [Balmino et al., 1973]; this could be because crustal thickening plays a large role in determining the center of figure for these planets, and the crust is not included in our model.

\section{Visual Comparison With Tomography: Effect of Flltering}

In order to make any comparisons between convection simulations and seismic models it is necessary to consider the effects of limited seismic resolution on the convective features. Although the filtering that seismic tomographic data sets and inversions perform on the actual Earth structure is complex, for the moment we approximate it by a simple truncation of the convection results at the nominal resolution of typical current tomographic models, i.e., $\ell=10$, Chebyshev degree $n=13$, and removal of the spherically symmetric $(\ell=0)$ component, although in the near future, more realistically filtered data sets may be possible [Johnson et al., 1993].

We show in other sections that even when filtered to seismic tomographic resolution, the effect of the phase change on globally averaged diagnostics of the thermal field is still clearly discernible, both in spectral heterogeneity maps (SHM) and in radial correlations between layers [Jordan et al., 1993]. The strong heterogeneity around the 670 (Figure 5a) which is associated with a peak in the radial profile of horizontally averaged heterogeneity [Peltier and Solheim, 1992; Tackley et al., 1993] is still visible at Chebyshev degree $n=13$ but disappears at $n=6$.

In the filtered temperature field for case 1 (Plate 7), all plumelike upwellings in the midmantle disappear completely.
The strongest of the broad cylindrical downwellings are visible in the MM as smeared out cold anomalies and thus may be discernible in tomographic models. Although the lateral heterogeneity spectrum in the upper mantle is dominated by long wavelengths, the most important convective features (the linear downwellings) are very narrow, and it is necessary to expand to at least $\ell \sim 31$ in order to see these features clearly, indicating the importance of obtaining seismic Earth models out to high spherical harmonic degree, even though most of the power has been demonstrated to be at long wavelengths [Tanimoto, 1990a; Su and Dziewonski, 1991].

There are some significant differences in deep mantle morphology between seismic Earth models and the convection simulations presented here. Seismic models indicate the existence of two major quasi-circular slow ("hot") regions at the base of the mantle underneath the Pacific and Africa, which are frequently interpreted as sources of hot upwelling plumes. These are surrounded by linear fast ("cold") regions situated approximately underneath past subduction zone locations [Fukao, 1992; Scrivner and Anderson, 1992; Richards and Engebretson, 1992]. In contrast, our models contain circular pools of cold material surrounded by hot ridges. Some convection simulations seem to demonstrate broad hot upwellings compatible with those seen in seismic tomography [e.g., Balachandar and Yuen, 1992]; however, a large fraction of basal heating is required for these broad plumes, which is not compatible with various geophysical constraints, as summarized by Stacey [1992].

If avalanches occur in Earth, these calculations indicate that the deep mantle would have a strongly depressed mean temperature, due to the efficient deposition of large volumes of cold material above the CMB. Calculation of actual temperature anomalies in the deep mantle from seismic tomography supports the hypothesis [Yuen et al., 1993]. When compared to this depressed mean temperature, hot anomalies in the DM appear much hotter than they actually are in absolute terms. This was shown in Plate 1, where large hot ridges are seen in the deep mantle. It can also be seen clearly by comparing the cross section in Plate 7, which has the $\ell=0$ term removed, with the unfiltered cross sections in Plate 5 . This effect may be important in the interpretation of seismic tomography, which is insensitive to the spherically symmetric temperature. The large, hot plumes which appear in the DM region of tomographic models may not be as hot in absolute terms as they appear relative to their surroundings. In the midmantle region, where our model predicts that the spherically averaged superadiabatic temperature is higher, their buoyancy relative to the immediate surroundings is lower, and they may not be able to rise to the upper mantle, as was seen in case 1 . This may explain why only tenuous structure is visible in the midmantle above these seemingly giant plumes. As discussed earlier, the departure of the geotherm from an adiabat is of similar magnitude to the rms temperature variations in the interior.

\section{Two-Point Correlation Functions}

It is desirable to develop ways of quantitatively comparing mantle flow models with seismic tomographic models, since visual comparisons can be somewhat subjective. Puster and Jordan [1994] proposed and tested a class of two-point autocorrelation functions to extract globally averaged characteristics from simulated or seismic data sets. Of these, Jordan et al. [1993] compare radial correlation functions 


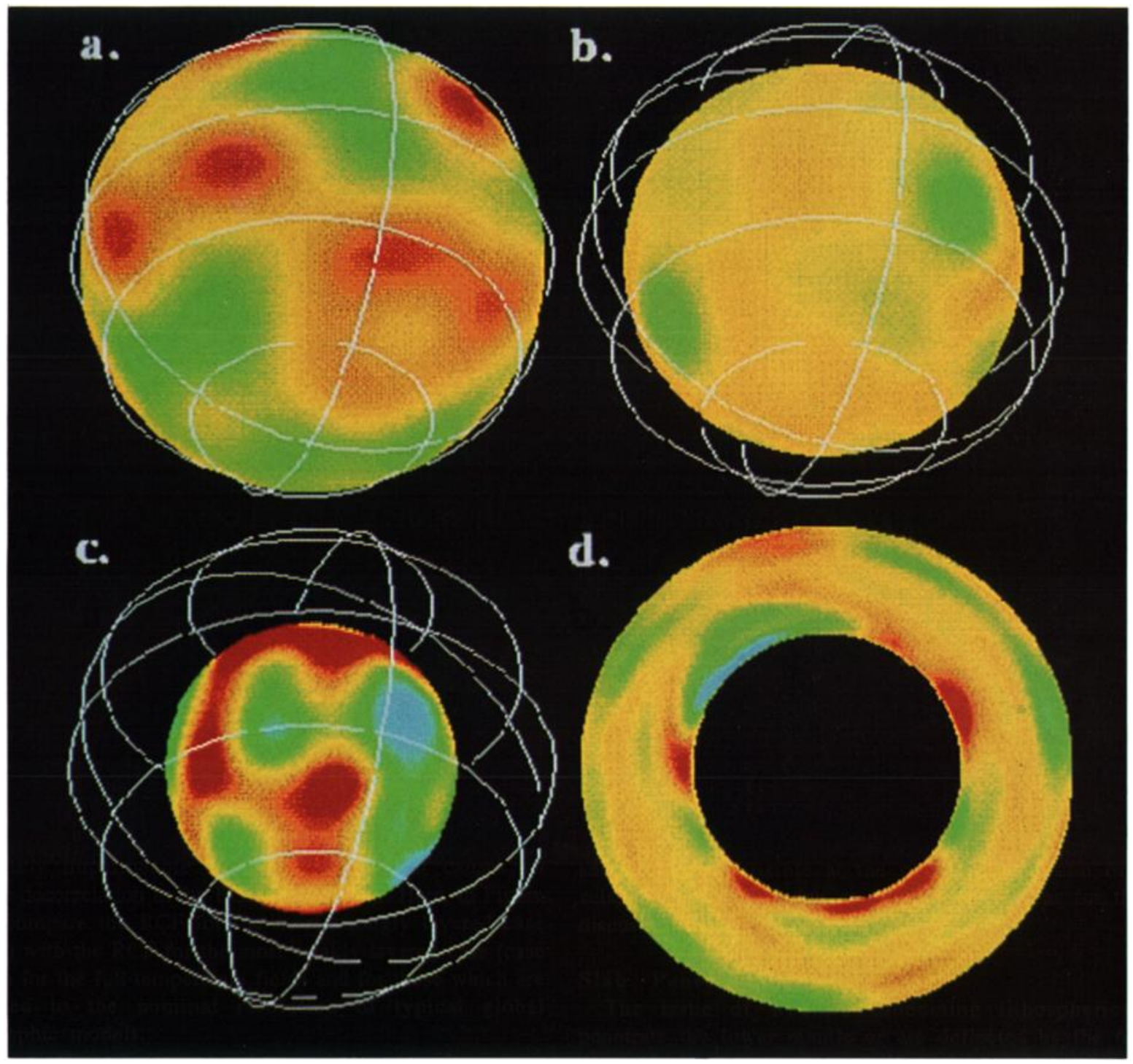

Plate 7. Effect of filtering on the temperature field for case 1. Field has been truncated to spherical harmonic degrees 1-10 and whole mantle Chebyshev degree 13. View is from same angle as Plate 3. (a) Shallow mantle, $200 \mathrm{~km}$ depth. (b) Midmantle, $1200 \mathrm{~km}$ depth. (c) Deep mantle, $2600 \mathrm{~km}$ depth. (d) Cross section.

(RCFs) for global seismic tomographic models, with those for frames from the phase change simulation of Tackley et al. [1993] and case 1 in this paper, finding the RCF to be a very sensitive detector of any flow stratification at $670 \mathrm{~km}$ depth, even at the nominal resolution of seismic tomography. Any stratification results in a decorrelation of structures across 670 km depth. Glatzmaier and Schubert [1993] show that a completely impermeable boundary at $670 \mathrm{~km}$ causes an extreme decorrelation in the RCF at this depth. Here, in Figure 7, we compare the RCF for the most strongly layered case (case 1) with the RCF for the most weakly layered case (case 4), both for the full temperature fields and for those which are truncated to the nominal resolution of typical global tomographic models.

In the unfiltered plots, the characteristic narrowing of the central correlation peak (running along the diagonal of the plots) at $670 \mathrm{~km}$ depth (nondimensional depth 0.23 ) is still clearly visible even for case 4, although the strong decorrelation between upper and lower mantles is less pronounced for that case. In the filtered plots, this narrowing is still clear in case 1 but less pronounced in case 4 . In case 1 , an additional constriction is visible in the lower mantle, indicating the boundary between regions $M M$ and DM.

RCFs for the current generation of seismic tomographic models do not, however, display any characteristics of flow stratification at $670 \mathrm{~km}$, even though such characteristics should still be visible at the nominal resolution of these seismic models [Jordan et al., 1993]. Reasons for this are discussed in the conclusions section.

\section{Slab Penetration}

The issue of whether subducting lithospheric slabs penetrate the lower mantle or are deflected by the $670-\mathrm{km}$ discontinuity is central to the question of mantle layering. The closest analogues to slabs in our model are the linear downwellings in the upper mantle, and thus our model results suggest that slabs do not immediately penetrate but instead build up in the transition zone until sufficient cold mass has 

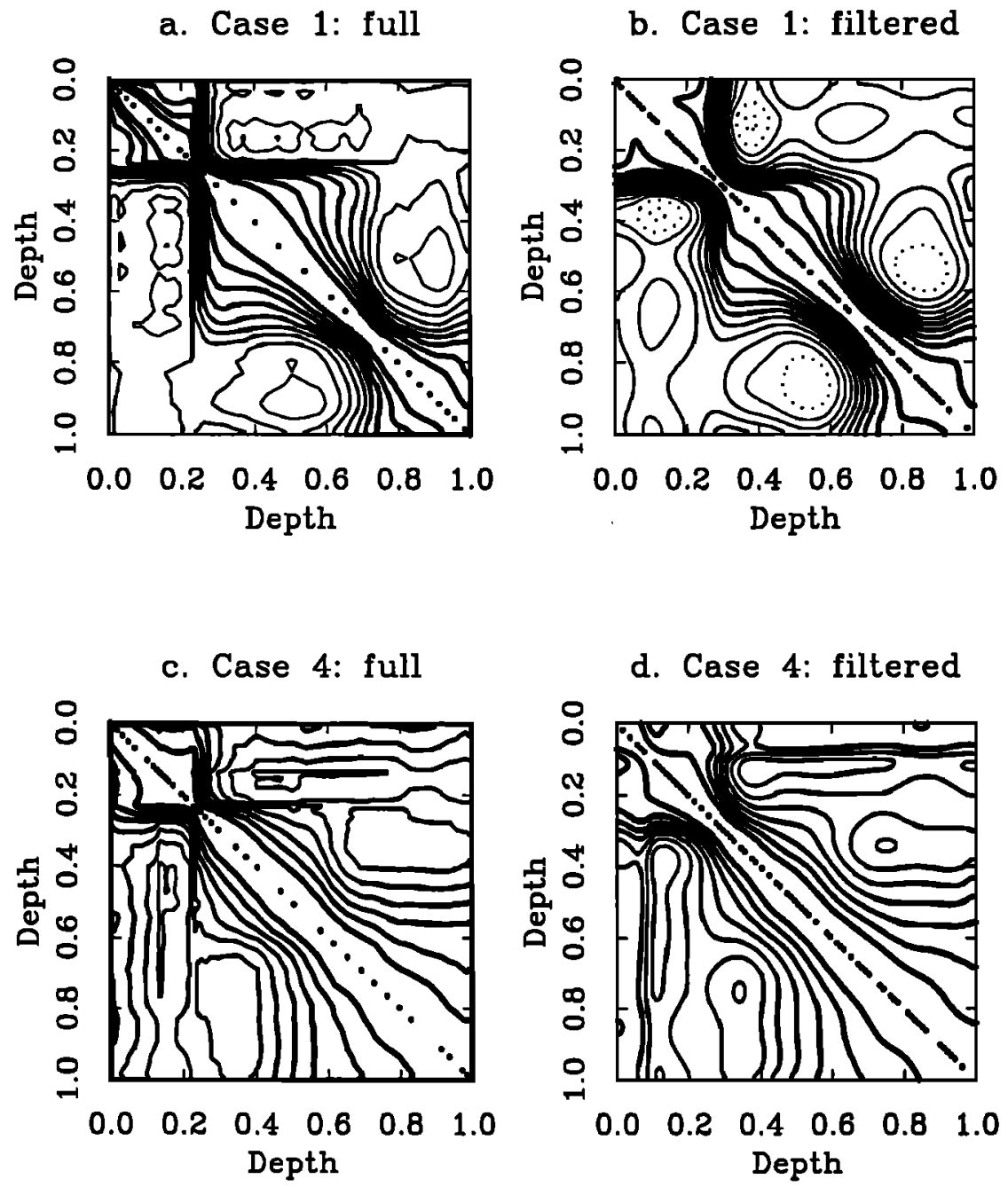

Figure 7. Radial correlation functions (RCF) for cases 1 and 4. (a) and (c) For the full entropy field, (b) and (d) for fields that have been truncated to the nominal resolution of typical seismic tomography (spherical harmonic degree 10, Chebyshev degree 13 using a whole mantle expansion).

accumulated to precipitate an avalanche into the lower mantle [Christensen and Yuen, 1985; Solheim and Peltier, 1994]. This picture would be consistent with (1) strong correlations between past locations of subduction and seismically fast ("cold") regions at the base of the upper mantle and top of the lower mantle [Scrivner and Anderson, 1992; Ray and Anderson, 1994], (2) recent tomography of slabs [Zhou and Clayton, 1990; van der Hilst et al., 1991; Fukao et al., 1992] showing that at least in some areas, slabs flatten out along the 670-km discontinuity and appear to stagnate in the transition zone, (3) recent global tomography [Su et al., 1992a] showing broad seismically fast areas at $600 \mathrm{~km}$ depth in places where subducted slabs would be expected to accumulate, and (4) global mapping of topography on the $660-\mathrm{km}$ discontinuity [Shearer and Masters, 1992] favoring models in which subducting slabs are deflected horizontally at the discontinuity.

However, the seismic evidence, reviewed by Lay [1994], does not paint a uniform picture, as slabs do appear to penetrate into the lower mantle in some regions [Creager and Jordan, 1984; Vidale and Garcia-Gonzalez, 1988; Fukao et al., 1992], and thus the true situation is probably complex, with some slabs penetrating and others being deflected at $670 \mathrm{~km}$ depth. Our model lacks temperature-dependent viscosity and hence the high viscosity of slabs (although slabs have warmed slightly by the time they reach $670 \mathrm{~km}$ depth), which may alter the results in two ways: (1) the overall planform and morphology of downwellings and avalanches and (2) the ability of a slablike downwelling to penetrate the 670 .

On the first point, we note that during a cylindrical avalanche in our models, cold material flows laterally along the "slabs" toward the avalanche, a mode of flow that is not possible when the slab is of much higher viscosity than its surroundings. Thus, in Earth, the supply of new cold material to an ongoing cylindrical avalanche is much more restricted, and the avalanche may therefore be shorter in duration. Indeed, junctions of linear downwellings, at which the large pools of cold material build up in our model, are not observed in Earth except at the intersection of the Pacific, Philippine, and Eurasia plates. If most of the buildups and avalanches are linear, this may make catastrophic breakthrough more difficult and more violent, as in two-dimensional models.

On the second point, simulations by Christensen and Yuen [1984] indicate that a viscous slab acts as a stress guide, penetrating the $670-\mathrm{km}$ phase boundary more easily. However, their model is strongly constrained in a manner that is likely 
to be most favorable to penetration: the slab is forced to be vertical, an angle of subduction not seen in Earth, and must bend back upon itself in order to deflect, a mode of deflection apparently not seen in Earth. In addition, no overall lateral movement of the slab or migration of the subduction zone was allowed. In contrast, laboratory experiments [Kincaid and Olson, 1987; Buttles et al., 1993] indicate that in the presence of retrograde trench migration [Garfunkel et al., 1986] and a viscosity jump at $670 \mathrm{~km}$, a highly viscous slab can flatten out along the $670-\mathrm{km}$ discontinuity without a phase change being necessary at all, provided there is a large enough viscosity contrast across 670 . In addition, the cold slab material is highly viscous and when deflected by the phase change has a greater tendency to stagnate in the transition zone [Kincaid and Olson, 1987; Gurnis and Hager, 1988; Buttles et al., 1993].

Any jumps in viscosity at $670 \mathrm{~km}$ depth, which could be a viscosity increase [Davies and Richards, 1992; Mitrovica and Peltier, 1993], viscosity decrease [Karato and $\mathrm{Li}, 1992$ ], or narrow zone of low viscosity [Forte et al., 1993] may serve to decouple flow in the upper and lower mantles [Ellsworth and Schubert, 1988], altering the likelihood of slab penetration.

\section{Other Possible Influences of Variable Viscosity}

Clearly, high priority must be given to incorporating temperature-dependent viscosity in convection models with phase changes. However, a viscosity which is dependent solely on temperature arguably makes such models less Earthlike than constant viscosity models. This is because the downwellings in models with purely temperature-dependent viscosity are broad and symmetric (i.e., two-sided) [Tackley, 1993; Steinbach and Yuen, 1993], with considerable stress occurring where they leave the upper boundary layer. Thus they do not resemble slabs in Earth, which are narrow and asymmetric (single-sided), and enter the interior easily due to subduction zone faults, thereby allowing them to transmit more stress onto any obstacle. The dynamics of a slab encountering the 670 may be significantly different from the dynamics of one of the former downwellings encountering the 670. Realistic Earth-like slabs are obtainable by imposing subduction zone "faults" at prescribed locations [Gurnis and Hager, 1988; Davies, 1989; Zhong and Gurnis, 1992; S.D. King and B.H. Hager, Subducted slabs and the geoid, 1, Numerical calculations with temperature-dependent viscosity, submitted to Journal of Geophysical Research, 1994] and much has been learned from such models. However, none of the above studies included phase changes; those that did [Christensen and Yuen, 1984; Zhong and Gurnis, 1994] have vertical, two-sided downwellings. In the long term it is desirable to generate a self-consistent (i.e., with no imposed features) model of variable-viscosity flow with phase changes, which must include some way of self-consistently generating plates and slabs, in itself a theoretical and numerical challenge that has so far eluded the geodynamical community.

The inclusion of temperature-dependent viscosity would result in a low viscosity layer immediately above the CMB due to the thermal boundary layer, which would strongly affect the formation and dynamics of upwelling plumes [Olson et al., 1987, 1993], making them narrower, more concentrated, more vigorous, possibly resulting in more of them, and allowing the formation of plume heads [Duncan and Richards, 1991]. However, the possible lack of realism in plume activity in our model is not of concern since on Earth, plumes are minor features, carrying only 10-15\% of the total heat flux [Davies and Richards, 1992; Sleep, 1990].

The addition of rigid plates and continents would probably reinforce the long wavelength nature of the flow [Davies, 1988; Gurnis and Zhong, 1991; Zhong and Gurnis, 1993]. The plate tectonic cycle, including subduction zone locations, may be controlled by the assembly and breakup of supercontinents [Gurnis, 1988], which would pump power into the very lowest spherical harmonic degrees [Anderson, 1982; Scrivner and Anderson, 1992].

\section{Conclusions}

We have provided a detailed analysis of numerical simulations of mantle convection which incorporate the major phase changes of the transition zone and investigated many ways in which the results can be compared to observations, such as seismic tomographic Earth models and the geoid. Although our model does not include the effects of variable viscosity, tectonic plates, or continents, many of the major characteristics of Earth are reproduced, such as the predominance of linear downwellings in the upper mantle (analagous to slabs) and the dominance of long-wavelength heterogeneity in the upper mantle and base of the mantle.

Our results indicate unambiguously that the effect of adding the exothermic phase change at $400 \mathrm{~km}$ depth is to diminish the propensity to layering by making avalanches smaller and more frequent. The system is much more sensitive to the strength of the $670-\mathrm{km}$ phase change than to the strength of the $400-\mathrm{km}$ phase change. Indeed, the current range of uncertainty in the Clapeyron slope at $670 \mathrm{~km}\left(-2\right.$ to $-6 \mathrm{MPa} \mathrm{K} \mathrm{K}^{-1}$ [Ito et al., 1990]) is likely to encompass a huge difference in model behavior.

Three distinct regions are evident, on the basis of both visual and globally averaged (e.g., spectral) characteristics. These are the upper mantle (UM), the midmantle (MM), and the deep mantle (DM). The upper mantle may be further subdivided into the transition zone, where the pooling of material occurs, and the shallow mantle. The UM and DM have large-amplitude long-wavelength signals, due to the pooling of cold downwellings at these depths, whereas the MM has the low-amplitude, broad spectrum typically associated with the interior of convecting regions. In the DM region, the pooling of cold avalanched material results in a zone of strongly depressed temperature, which may partially explain the appearance of large, seemingly very hot regions at this depth in seismic Earth models.

The geoid amplitude and spectrum are not significantly affected by the phase change induced layering and are similar to those of Earth, Venus, and simulations with no phase change. This is because the contribution from the heterogeneity introduced into the transition zone is balanced by an opposite and nearly equal contribution from deflection of the $670-\mathrm{km}$ discontinuity. The geoid is strongly influenced by structure the upper mantle and is weakly influenced by midmantle structure. Avalanches may be visible as geoid lows. However, the sign of the geoid anomaly over downwellings is opposite to that of Earth. The most likely explanation for Earth's behavior is a large viscosity increase at around $670 \mathrm{~km}$ depth. Further models including an appropriate viscosity increase will be needed to examine the nature of the circulation and layering for models with realistic geoids.

In order to make comparisons with seismic Earth 
observations we have looked at diagnostics based on the thermal field (spectral heterogeneity maps and radial correlation functions) and shown that these indicators are very sensitive, even at seismic tomographic resolution, to quite small amounts of stratification. Diagnostics based on the long-wavelength velocity field, however (such as radial mass flux), are rather insensitive to the degree of layering occurring in our model, due to the wavelength dependence of flow stratification: At long wavelengths, whole mantle flow is observed, whereas short wavelengths display flow stratification due to the modulation of downwellings in the transition zone.

This observation that the long-wavelength thermal field is strongly affected by the phase transition, whereas long-wavelength flow field is not, may appear contradictory, since the thermal and flow fields are coupled in a convecting system and most of the heat transport across the phase boundaries is by advection. It is not surprising that long wavelengths of flow penetrate the 670 , since these long wavelengths are sensitive only to structure that is vertically averaged over a large lengthscale similar to the horizontal wavelength. The question then is: how can long-wavelength thermal structure build up about the 670 if the flow field goes through? There are two points to make here. One is that the long wavelengths in the TZ thermal field arise not through direct inhibition of long-wavelength thermal structures but rather through a cascade of power from short wavelengths (a narrow downwelling encountering the phase transition) to longer wavelengths (as the downwelling pools). The second is that the spectral content of velocity field will be a highly filtered (both radially and azimuthally) version of the thermal field, with a 1/wavelength ${ }^{2}$ relationship expected from simple scaling analysis of the momentum equation. Thus, even if the longwavelength thermal field changes abruptly at 670 , the longwavelength velocity field would not. Combining these two points, one can understand the sequence of events at a particular azimuthal location: (1) a downwelling encounters the 670 and pools. Long-wavelength flow is inhibited at all radii, as a broad pool accumulates in the $T Z$ until (2) the point of criticality is passed and an avalanche occurs.

Actual comparisons with Earth observations appear contradictory. The characteristics of the lateral spectrum at different depths (shown by spectral heterogeneity maps), and in particular the identification of three distinct regions in the mantle, are compatible with seismic tomographic models, particularly that of $S u$ et al. [1992b]. However, there is no evidence of the peak in the globally averaged lateral heterogeneity amplitude at $670 \mathrm{~km}$ depth which would be expected, even for weak layering [Peltier and Solheim, 1992; Tackley et al., 1993]. In addition, there is no evidence in the radial correlation functions (RCFs) of current generation seismic models, for any flow stratification at $670 \mathrm{~km}$ [Jordan et al., 1993].

Since we have demonstrated that such diagnostics based on the thermal field are very sensitive to small degrees of stratification, even when filtered to the nominal resolution of seismic tomography, it would be tempting to conclude that Earth does not currently exhibit any significant amount of flow stratification. However, such a conclusion would be contradicted by other forms of more localized evidence, such as tomographic images of slabs being deflected along the 670km discontinuity [van der Hilst et al., 1991; Fukao et al., 1992]. It could be that there are inherent limitations in the data, parameterization, or inversion techniques used in current seismic models which would mask characteristics such as a decorrelation in the RCF. Alternatively, it could be that future, more realistic convection models will display a mode of stratification that is not detected in these diagnostics. It has been suggested that Earth may flip in a globally synchronous manner between layered and whole mantle modes of flow, with it currently exhibiting a whole mantle state [Peltier and Solheim, 1993]. However, we have argued that such global synchronicity is unlikely in three dimensions. In addition, there is no evidence in geological data for a recent massive acceleration in surface plate velocities that would be expected to accompany such a global transition [Weinstein, 1993].

In any case, reconciling both globally averaged and local characteristics of mantle convection simulations with observations of Earth and Venus is an important challenge for the future.

Acknowledgments. We thank D.A. Yuen and W.R. Peltier for preprints and useful discussions, S.D. King and W.S. Kiefer for constructive reviews, and W.M. Kaula for providing us with planetary gravity coefficients. This research was performed in part using the Intel Touchstone Delta operated by Caltech on behalf of the Concument Supercomputing Consortium. Access to this facility was provided by Caltech. This work was supported by NSF grant EAR9017893 and by the Institute of Geophysics and Planetary Physics at Los Alamos. Division of Geological and Planetary Sciences contribution 5331 .

\section{References}

Akaogi, M., and E. Ito, Refinement of enthalpy measurement of $\mathrm{MgSiO}_{3}$ perovskite and negative pressure-temperature slopes for perovskite-forming reactions, Geophys. Res. Lett., 20, 1839 1842, 1993.

Akaogi, M., E. Ito, and A. Navrotsky, Olivine-modified spinel-spinel transitions in the system $\mathrm{Mg}_{2} \mathrm{SiO}_{4}-\mathrm{Fe}_{2} \mathrm{SiO}_{4}$ : Calorimetric measurements, thermochemical calculation, and geophysical application, J. Geophys. Res., 94, 15671-15685, 1989.

Anderson, D.L., Hotspots, polar wander, mesozoic convection, and the geoid, Nature, 297, 391-393, 1982.

Anderson, D.L., A seismic equation of state, II, Shear properties and thermodynamics of the lower mantle, Phys. Earth Planet. Inter. 45, 307-323, 1987.

Anderson, D.L., Theory of the Earth, 366 pp, Blackwell, Boston, Mass., 1989.

Anderson, O.L., H. Oda, and D. Isaak, A model for the computation of thermal expansivity at high compression and high temperatures: MGO as an example, Geophys. Res. Lett., 19, 1987-1990, 1992.

Balachandar, S., and D.A. Yuen, Time-dependent three-dimen sional compressible convection with depth-dependent properties, Geophys. Res. Lett., 19, 2247-2250, 1992.

Balmino, G., K. Lambeck, and W.M. Kaula, A spherical harmonic analysis of the Earth's topography, J. Geophys. Res., 78, 478481, 1973.

Baumgardner, J.R., Three-dimensional treatment of convective flow in the Earth's mantle, J. Stat. Phys., 39, 501-511, 1985.

Baumgardner, J.R., Application of supercomputers to 3-D mantle convection, in The Physics of the Planets, edited by S.K. Runcom, pp. 199-231, John Wiley, New York, 1988.

Benz, H.M., and J.E. Vidale, Sharpness of upper-mantle dis continuities determined from high-frequency reflections, Nature, 365, 147-150, 1993.

Bercovici, D., G. Schubert, and G. Glatzmaier, Three-dimensional spherical models of convection in the Earth's mantle, Science, 244, 950-955, 1989. 
Bercovici, D., G. Schubert, and P.J. Tackley, On the penetration of the $660 \mathrm{~km}$ phase change by mantle downflows, Geophys. Res. Lett, 20, 2599-2602, 1993.

Birch, F., Elasticity and composition of the Earth's interior, $J$. Geophys. Res., 57, 227-286, 1952.

Breuer, D., and T. Spohn, Cooling of the Earth, Urey ratios, and the problem of potassium in the core, Geophys. Res. Lett., 20, 16551658, 1993

Busse, F.H., Fundamentals of thermal convection, in Mantle Convection: Plate Tectonics and Global Dynamics, edited by W.R. Peltier, pp. 23-95, Gordon and Breach, New York, 1989.

Buttles, J., L. Gillou and P. Olson, Laboratory experiments on the deep structure of subducted slabs (abstract), Eos Trans. AGU, 74(16), Spring Meeting Suppl., 300, 1993.

Chopelas, A., and R. Boehler, Thermal expansivity in the lower mantle, Geophys. Res. Lett., 19, 1983-1986, 1992.

Christensen, U.R., and D.A. Yuen, The interaction of a subducting lithospheric slab with a chemical or phase boundary, J. Geophys. Res., 89, 4389-4402, 1984.

Christensen, U.R., and D.A. Yuen, Layered convection induced by phase transitions, J. Geophys. Res., 90, 10,291-10,300, 1985.

Christensen, U., and D. Yuen, On the aspect ratio of Rayleigh-Benard convection cells, Geophys. Res. Lett., 15, 597-600, 1988.

Creager, K.C., and T.H. Jordan, Slab penetration into the lower mantle, J. Geophys. Res., 89, 3031-3049, 1984.

Davies, G.F., Role of the lithosphere in mantle convection, $J$. Geophys. Res., 93, 10,451-10,466, 1988.

Davies, G.F., Mantle convection with a dynamic plate: Topography, heat flow and gravity anomalies, Geophys. J. Int., 98, 461-464, 1989.

Davies, G.F., and M.A. Richards, Mantle convection, J. Geol., 100, 151-206, 1992.

Davies, J.H., O. Gudmundsson, and R.W. Clayton, Spectra of mantle shear velocity structure, Geophys. J. Int., 108, 865-882, 1992.

Duffy, T.S., and T.J. Ahrens, Thermal expansion of mantle and core materials at very high pressures, Geophys. Res. Lett., 20, 11031106, 1993.

Duncan, R.A., and M.A. Richards, Hotspots, mantle plumes, flood basalts, and true polar wander, Rev. Geophys., 29, 31-50, 1991.

Dziewonski, A.M. and D.L. Anderson, Preliminary reference Earth model, Phys. Earth Planet. Inter., 25, 297-356, 1981.

Ellsworth, K., and G. Schubert, Numerical models of thermally and mechanically coupled two-layer convection of highly viscous fluids, Geophys. J., 93, 347-363, 1988.

Forte, A.M., A.M. Dziewonski, and R.L. Woodward, Aspherical structure of the mantle, tectonic plate motions, nonhydrostatic geoid, and topography of the core-mantle-boundary, in Dynamics of the Earth's Deep Interior and Earth Rotation, Geophys. Monogr. Ser., vol. 72, edited by J.L. LeMouel, D.E. Smylie, and T. Herring, AGU, Washinton, D.C., 1993.

Fukao, Y., M. Obayashi, H. Inoue, and M. Nenbau, Subducting slabs stagnant in the mantle transition zone, J. Geophys. Res., 97, 4809-4822, 1992.

Fukao, Y., Seismic tomogram of the Earth's mantle: Geodynamic implications, Science, 258, 625-630, 1992.

Garfunkel, Z., C.A. Anderson, and G. Schubert, Mantle circulation and the lateral migration of subducted slabs, J. Geophys. Res., 91, 7205-7223, 1986.

Glatzmaier, G.A., Numerical simulations of mantle convection: Timedependent, three-dimensional, compressible, spherical shell, Geophys. Astrophys. Fluid Dyn., 43, 223-264, 1988.

Glatzmaier, G.A., G. Schubert, and D. Bercovici, Chaotic, subductionlike downflows in a spherical model of convection in the Earth's mantle, Nature, 347, 274-277,1990.

Glatzmaier, G.A., and G. Schubert, Three-dimensional spherical models of layered and whole mantle convection, J. Geophys. Res., 98, 21,969-21,976, 1993.

Gudmundsson, O., J.H. Davies, and R.W. Clayton, Stochastic analysis of global traveltime data: Mantle heterogeneity and random errors in the ISC data, Geophys. J. Int., 102, 25-43, 1990.

Gurnis, M., Large-scale mantle convection and the aggregation and dispersal of supercontinents, Nature, 332, 695-699, 1988.

Gurnis, M., and B.H. Hager, Controls on the structure of subducted slabs, Nature, 335, 317-321, 1988.

Gurnis, M., and S. Zhong, Generation of long wavelength heterogeneity in the mantle by the dynamic interaction between plates and convection, Geophys. Res. Lett., 18, 581-584, 1991.

Hager, B.H. and R.W. Clayton, Constraints on the structure of mantle convection using seismic observations, flow models, and the geoid, in Mantle Convection: Plate Tectonics and Global Dynamics, edited by W.R. Peltier, PP. 657-764, Gordon and Breach, New York, 1989.

Hager, B.H., and M.A. Richards, Long-wavelength variations in Earth's geoid: Physical models and dynamical implications, Philos. Trans. R. Soc. London, 328, 309-327, 1989.

Honda, S., D.A. Yuen, S. Balachandar, and D. Reuteler, Threedimensional instabilities of mantle convection with multiple phase transitions, Science, 259, 1308-1311, 1993.

Houseman, G., The dependence of convection planform on mode of heating, Nature, 332, 346-349, 1988.

Inoue, H., Y. Fukao, K. Tanabe, and Y. Ogata, Whole mantle $P$-wave travel time tomography, Phys. Earth Planet. Inter., 59, 294-328, 1990.

Ito, E., and E. Takahashi, Postspinel transformations in the system $\mathrm{Mg}_{2} \mathrm{SiO}_{4}-\mathrm{Fe}_{2} \mathrm{SiO}_{4}$ and some geophysical implications, J. Geophys. Res., 94, 10,637-10,646, 1989.

Ito, E., M. Akaogi., L. Topor, and A. Navrotsky, Negative pressuretemperature slopes for reactions forming $\mathrm{MgSiO}_{3}$ perovskite from calorimetry, Science, 249, 1275-1278, 1990.

Jarvis, G.T., and W.R. Peltier, Lateral heterogeneity in the convecting mantle, J. Geophys. Res. 91, 435-451, 1986.

Jeanloz, R., Effects of phase transitions and possible compositional changes on the seismological structure near $650 \mathrm{~km}$ depth, Geophys. Res. Lett., 18, 1743-1746, 1991.

Johnson, S., G. Masters, P.J. Tackley, and G.A. Glatzmaier, How well can we resolve a convecting Earth with seismic data? (abstract), Eos Trans. AGU, 74(43), Fall Meeting suppl., 80, 1993.

Jordan, T.H., P. Puster, G.A. Glatzmaier, and P.J. Tackley, Comparisons of seismic Earth models and mantle flow models using radial correlation functions, Science, 261, 1427-1431, 1993.

Karato, S., Importance of anelasticity in the interpretation of seismic tomography, Geophys. Res. Lett., 20, 1623-1626, 1993.

Karato, S., and P. Li, Diffusion creep in perovskite-Implications for the rheology of the lower mantle, Science, 255, 1238-1240, 1992.

Katsura, T., and $\mathrm{E}$. Ito, The system $\mathrm{Mg}_{2} \mathrm{SiO}_{4}-\mathrm{Fe}_{2} \mathrm{SiO}_{4}$ at high pressures and temperatures: Precise determination of stabilities of olivine, modified spinel, and spinel, J. Geophys. Res., 94, 15,663-15,670, 1989.

Kaula, W.M., Gravity fields: Implications for planetary interiors, in Encyclopedia of Geophysics, edited by D.E. James, pp. 622-627, Van Nostrand Reinhold, New York, 1989.

Kincaid, C., and P. Olson, An experimental study of subduction and slab migration, J. Geophys. Res., 92, 13,832-13,840, 1987.

King, S.D., and G. Masters, An inversion for radial viscosity structure using seismic tomography, Geophys. Res. Lett., 19, 1551-1554, 1992.

Lay, T., The fate of descending slabs, Annu. Rev. Earth Planet. Sci, 22, 33-62, 1994.

Leitch, A., and D. Yuen, Internal heating and thermal constraints on the mantle, Geophys. Res. Lett., 16, 1407-1410, 1989.

Liu, M., D.A. Yuen, W. Zhao, and S. Honda, Development of diapiric structures in the upper mantle due to phase transitions, Science, 252, 1836-1839, 1991.

Loper, D.E., and F.D. Stacey, The dynamical and thermal structure of deep mantle plumes, Phys. Earth Planet. Inter., 33, 304-317, 1983.

Machetel, P., and P. Weber, Intermittent layered convection in a 
model mantle with an endothermic phase change at $670 \mathrm{~km}$, Nature, 350, 55-57, 1991.

Masters, T.G., H. Bolton, and P. Shearer, Large-scale 3-dimensional structure of the mantle, Eos Trans. AGU, 73(14), Spring Meeting suppl., 201, 1992.

Mitrovica, J.X., and W.R. Peltier, The inference of mantle viscosity from an inversion of the Fennoscandian relaxation data, Geophys. J. Int., 114, 45-62, 1993.

Nakanishi, I., and D.L. Anderson, Measurements of mantle wave velocities and inversion for lateral heterogeneity and anisotropy, 1, Analysis of great-circle phase velocities, J. Geophys. Res., 88, 10,267-10,283, 1983.

Olson, P., G. Schubert, and C. Anderson, plumes formation in the D" layer and the roughness of the core-mantle boundary, Nature, 327, 409-413, 1987.

Olson, P., G. Schubert, and C. Anderson, Structure of axisymmetric mantle plumes, J. Geophys. Res., 98, 6829-6844, 1993.

Osako, M., and E. Ito, Thermal diffusivity of $\mathrm{MgSiO}_{3}$ perovskite, Geophys. Res. Lett., 18, 239-242, 1991.

Parmentier, E.M., C. Sotin and B.J. Travis, Turbulent 3-D thermal convection in an infinite Prandil number, volumetrically heated fluid: Implications for mantle dynamics, Geophys. J. Int., 116, 241-251, 1994.

Peltier, W.R., and L.P. Solheim, Mantle phase transitions and layered chaotic convection, Geophys. Res. Lett., 19, 432-324, 1992.

Peltier, W.R., and L.P Solheim, The supercontinent cycle and phase transition modulated mantle mixing (abstract), Eos Trans. AGU, 74(16), Spring Meeting suppl., 47, 1993.

Phipps Morgan, J., and P.M. Shearer, Seismic constraints on flow and discontinuity topography near $660 \mathrm{~km}-\mathrm{New}$ evidence for whole mantle convection, Nature, 365, 506-511, 1993.

Puster, P., and T.H. Jordan, Stochastic analysis of mantle convection experiments using two-point correlation functions, Geophys. Res. Lett., 21, 305-308, 1994.

Rapp, R.H., and N.K. Pavlis, The development and analysis of geopotential coefficient models to spherical harmonic degree 360 , J. Geophys. Res., 95, 21,885-21,911, 1990.

Ray, T.W., and D.L. Anderson, Spherical disharmonics in the Earth sciences and the spatial solution: Ridges, hotspots, slabs, geochemistry, and tomography correlations, J. Geophys. Res., 99 , 9605-9614, 1994.

Ricard, Y., and B. Wuming, Inferring the viscosity and 3-D density structure of the mantle from geoid, topography and plate velocities, Geophys. J. Int., 105, 561-571, 1991.

Ricard, Y., L. Fleitout, and C. Froidevaux, Geoid heights and lithospheric stresses for a dynamic Earth, Ann. Geophys., 2, 267 286, 1984.

Richards, M.A., and D.C. Engebretson, Large scale mantle convection and the history of subduction, Nature, 355, 437-440, 1992.

Richards, M.A., and B.H. Hager, Geoid anomalies in a dynamic Earth, J. Geophys. Res., 89, 5987-6002, 1984.

Ringwood, A.E., Phase transformations and differentiation in subducted lithosphere: Implications for mantle dynamics, basalt petrogenesis, and crustal evolution, J. Geol., 90, 611-643, 1982.

Ringwood, A.E., Phase transformations and their bearing on the constitution and dynamics of the mantle, Geochim. Cosmochim. Acta, 55, 2083-2110, 1991.

Schubert, G., Subsolidus convection in the mantles of terrestrial planets, Annu. Rev. Earth. Planet. Sci., 7, 289-342, 1979.

Schubert, G., Numerical models of mantle convection, Annu. Rev. Fluid Mech., 24, 359-394, 1992.

Schubert, G., and D.L. Turcotte, Phase transitions and mantle convection, J. Geophys. Res., 76, 1424-1432, 1971.

Schubert, G., D.A. Yuen, and D.L. Turcotte, Role of phase transitions in a dynamic mantle, Geophys. J. R. Astron. Soc., 42, 705-735, 1975.

Schubert, G., D.J. Stevenson, and P. Cassen, Whole planet cooling and the radiogenic heat source contents of the Earth and Moon, $J$. Geophys. Res., 85, 2531-2538, 1980.
Schubert, G., D. Bercovici, and G.A. Glatzmaier, Mantle dy namics in Mars and Venus: Influence of an immobile lithosphere on threedimensional mantle convection, J. Geophys. Res., 95, 14,10514,129, 1990.

Scrivner, C., and D.L. Anderson, The effect of post Pangea subduction on global mantle tomography and convection, Geophys. Res. Lett., 19, 1053-1056, 1992.

Sharpe, H.N., and W.R. Peltier, Parameterized mantle convection and the Earth's thermal history, Geophys. Res. Lett., 5, 737-740, 1978.

Shearer, P.M., and T.G. Masters, Global mapping of topography on the 660-km discontinuity, Nature, 355, 791-796, 1992.

Sleep, N.H., Hotspots and mantle plumes: Some phenomenology, $J$. Geophys. Res., 95, 6715-6736, 1990.

Solheim, L.P., and W.R. Peltier, Avalanche effects in phase transition modulated thermal convection: A model of the Earth's mantle, J. Geophys. Res., 99, 6997-7018, 1994.

Stacey, F., Physics of the Earth, 3rd Ed., 513 pp., Brookfield, Kenmore, Queensland, Australia, 1992.

Steinbach, V., and D.A. Yuen, The effects of multiple phase transitions on Venusian mantle convection, Geophys. Res. Lett., 19. 2243-2246, 1992.

Steinbach, V., and D.A. Yuen, Convection with phase transitions and temperature-dependent viscosity (abstract), Eos Trans. AGU., 74(16), Spring Meeting suppl., 300, 1993.

Steinbach, V., D.A. Yuen, and W.L. Zhao, Instabilities from phasetransitions and the timescales of mantle thermal evolution, Geophys. Res. Lett., 20, 1119-1122, 1993.

Su, W.-J., and A.M. Dziewonski, Predominance of long-wavelength heterogeneity in the mantle, Nature, 352, 121-126, 1991.

Su, W.-J., and A.M. Dziewonski, On the scale of mantle heterogeneity, Phys. Earth. Planet. Inter., 74, 29-54, 1992.

Su,W.-J., R.L. Woodward, and A.M. Dziewonski, Deep origin of modocean-ridge seismic velocity anomalies, Nature, 360, 149-152, 1992a.

Su, W.-J., R.L. Woodward, and A.M. Dziewonski, Joint inver sions of travel-time and waveform data for 3-D models of the Earth up to degree 12 (abstract), Eos Trans. AGU., 73 (14), Spring Meeting suppl., 201-202, 1992b.

Tackley, P.J., D.J. Stevenson, G.A. Glatzmaier, and G. Schubert, Effects of an endothermic phase transition at $670 \mathrm{~km}$ depth in a spherical model of convection in the Earth's mantle, Nature, 361, 699.704, 1993.

Tackley, P.J., Effects of strongly temperature-dependent viscosity on time-dependent, three-dimensional models of mantle convection, Geophys. Res. Lett., 20, 2317-2320, 1993.

Tanimoto, T., Predominance of large-scale heterogeneity and the shift of velocity anomalies between the upper and lower mantle, J. Phys. Earth, 38, 493-509, 1990a.

Tanimoto, T., Long-wavelength $S$-wave velocity structure throughout the mantle, Geophys. J. Int., 100, 327-336, $1990 \mathrm{~b}$.

Travis, B., S. Weinstein, and P. Olson, Three-dimensional convection planforms with internal heat generation, Geophys. Res. Lett., 17, 243-246, 1990.

Turcotte, D.L., and G. Schubert, Geodynamics: Applications of Continuum Physics to Geological Problems, pp. 279-285, John Wiley, New York, 1982.

van der Hilst, R., R. Engdahl, W. Spakman, and G. Nolet, Tomographic imaging of subducted lithosphere below north west Pacific island arcs, Nature, 353, 37-43, 1991.

Vidale, J.E., and D. Garcia-Gonzales, Seismic observation of a highvelocity slab $1200-1600 \mathrm{~km}$ in depth, Geophys. Res. Lett., 15 , 369-372, 1988.

Weinstein, S.A., Induced compositional layering in a convecting fluid layer by an endothermic phase-transition, Earth Planet. Sci. Lett., 113, 23-39, 1992.

Weinstein, S.A., Catastrophic overturn of the Earth's mantle driven by multiple phase changes and internal heat generation, Geophys. Res. Lett., 20, 101-104, 1993. 
Wicks, C.W., and M.A. Richards, A detailed map of the $660-\mathrm{km}$ discontinuity beneath the Izu-Bonin subduction zone, Science, 261, 1424-1427, 1993.

Wyllie, P.J., Magma genesis, plate tectonics, and chemical differentiation of the Earth, Rev. Geophys., 26, 370-404, 1988.

Yuen, D.A., O. Cadek, A. Chopelas and C. Matyska, Geophysical inferences of thermal-chemical structures in the lower mantle, Geophys. Res. Lett., 20, 899-902, 1993.

Yuen, D.A., D.M. Reuteler, S. Balachandar, V. Steinbach, A.V. Malevsky, and J.L. Smedsmo, Various influences on threedimensional mantle convection with phase transitions, Phys. Earth Planet. Inter., in press, 1994.

Zhao, W., D.A. Yuen, and S. Honda, Multiple phase transitions and the style of mantle convection, Phys. Earth Plan. Inter., 72, 185$210,1992$.

Zhong, S., and M. Gurnis, Viscous flow model of a subduction zone with a faulted lithosphere: Long and short wavelength topography, gravity and geoid, Geophys. Res. Lett., 19, 1891-1894, 1992.

Zhong, S. and M. Gurnis, Dynamic feedback between a continentlike raft and thermal convection, J. Geophys. Res., 98, 12,21912,232, 1993.

Zhong, S. and M. Gurnis, Role of plates and temperature-dependent viscosity in phase change dynamics, J. Geophys. Res., in press, 1994.
Zhou, H.W., and D.L. Anderson, Search for deep slabs in the northwest Pacific mantle, Proc. Natl. Acad. Sci. U. S. A. , 86, 8602-8606, 1989.

Zhou, H.W., and R.W. Clayton, $P$ and $S$ wave travel time inversions for subducting slab under the island arcs of the northwest Pacific, $J$. Geophys. Res., 95, 6829-6851, 1990.

G.A. Glatzmaier, Institute of Geophysics and Planetary Physics, Los Alamos National Laboratory, Los Alamos, NM 87545. (e-mail: gag @lanl.gov)

G. Schubert, Department of Earth and Space Sciences, University of California Los Angeles, Los Angeles, CA 90024. (e-mail: gschubert @geovax.ess.ucla.edu)

D.J. Stevenson, Division of Geological and Planetary Sciences, California Institute of Technology, Pasadena, CA 91125. (e-mail: djs@arms.gps.caltech.edu)

P.J. Tackley, Seismological Laboratory, California Institute of Technology, Pasadena, CA 91125 . (e-mail: paul@seismo.gps.caltech.edu)

(Received September 20, 1993; revised March 14, 1994; accepted March 31, 1994.) 